\title{
Ein Strafgericht für die Khmer Rouge. Herausforderung für Kambodscha und das Völkerstrafrecht
}

\author{
Von Jörg Menzel, Phnom Penh
}

Über siebenundzwanzig Jahre nachdem die so genannten Khmer Rouge unter ihrem Führer Saloth Sar (Pol Pot) von einer vietnamesischen Invasionsarmee aus ihrer knapp vierjährigen Macht in Kambodscha gejagt wurden, haben am 3. Juli 2006 mit der Vereidigung von Richtern und Staatsanwälten die Vorbereitungen zur Einrichtung eines gemischt nationalinternationalen Gerichts, das den noch lebenden Hauptverantwortlichen dieses Regimes den Prozess machen soll, einen Abschluss gefunden. Die Regierung der Roten Khmer hat, gemessen an der Bevölkerungszahl, in ihrer kurzen Herrschaftszeit wohl mehr Tote in der eigenen Bevölkerung zu verantworten als jede andere Staatsführung des an Brutalitäten nicht armen 20. Jahrhunderts. Kambodscha ist noch heute ein Staat, der im Bewusstsein der Menschheit fast unmittelbar mit ultimativem Staatsverbrechen in Verbindung gebracht wird. Natürlich ist da die Ruinenstadt „Angkor“, Sinnbild einer großen Vergangenheit dieses Volkes und wiederbelebte Touristenattraktion im verdienten Rang eines der größten archäologischen Schätze der Welt, aber die aktuelle internationale Wahrnehmung ist nach wie vor geprägt von dem Staatsexzess in der zweiten Hälfte der 1970er Jahre. Es scheint, als stünden nun Strafverfahren für zumindest einige der noch lebenden und mit einer Ausnahme bislang in Freiheit lebenden Haupttäter dieses Regimes bevor. Das speziell hierfür einzurichtende Gericht wird, auf nationalen und internationalen Rechtsgrundlagen basierend, aus nationalen und internationalen Richtern bestehen. Es stellt damit gleichermaßen eine Herausforderung für das kambodschanische Recht wie für das Völkerstrafrecht dar.

\section{Die Verbrechen der Khmer Rouge}

Als die Roten Khmer im April 1975 mit dem Einmarsch in die Hauptstadt Phnom Penh in Kambodscha vollständig die Macht übernahmen, hatte das Land bereits einen langen und gewaltvollen Krieg hinter sich. Innerer Bürgerkrieg und Verstrickung in die Auseinandersetzungen um das benachbarte Vietnam hatten ungezählte Todesopfer gefordert. Zwischen 1965 und 1973 waren weite Landgebiete Kambodschas flächendeckend von den USA nach neueren Schätzungen mit mehr Bomben belegt worden als im gesamten Zweiten Weltkrieg von den USA abgeworfen wurden. In dieser Zeit wurden nicht nur vielleicht ca. 600.000 
Kambodschaner getötet und etwa 2 Millionen Binnenflüchtlinge in die Städte getrieben ${ }^{1}$, sondern auch den sich radikalisierenden Khmer Rouge unzählige neue Kämpfer in die Arme getrieben ${ }^{2}$. Selbst Gegner der Roten Khmer nahmen deren Sieg den Berichten nach im ersten Augenblick bisweilen mit Erleichterung auf, konnte man sich doch nun zumindest ein Ende der Kämpfe erhoffen. Die neuen Machthaber freilich fackelten nicht einen Augenblick, das Land in neue - beispiellose - Leidenszeit zu stürzen ${ }^{3}$. Dem Einmarsch folgte die unmittelbare und systematische Exekution der Funktionäre des bisherigen Regimes sowie die vollständige Evakuierung der Hauptstadt sowie sonstiger großer Städte im Land. Zur Bezeichnung des Regimes wurde in Deutschland der Begriff des „Steinzeitkommunismus“" gebräuchlich und es liegt auf der Hand, dass das Gedankengut der Khmer Rouge eine historisch einzigartige Übersteigerung kommunistischer Ideologie und mehr spezifisch der maoistischen Kulturrevolution ist. Zwar haben sich die Führer dieses Regimes nie „theoretisch“ erklärt, Saloth Sahr, der unter seinem Kriegsnamen Pol Pot berühmt wurde, sowie seine Kollegen waren aber durchaus gut gebildet, hatten zum Teil in Frankreich studiert (Pol Pot selbst allerdings ohne „Abschluss“") ${ }^{4}$. Freilich verloren sie offenbar im Verlauf des auch von der Gegenseite in aller Brutalität geführten Dschungelkrieges die Hemmnisse eines zivilisatorischen Gewissens ${ }^{5}$. Kennzeichen der Politik der Roten Khmer waren die vollständige Kollektivierung und Agrarisierung der Bevölkerung, Auflösung traditioneller Familienstrukturen, Trennung von Eltern und Kindern, Zwangs-

So die Ergebnisse einer finnischen Untersuchung, vgl. Kimmo Kiljunen (ed.), Kampuchea. Decade of Genocide. Report of a Finnish Inquiry Commission, 1984, S. 5 f. Nachweis weiterer Schätzungen bei Nicole Barrett, Holding Individual Leaders Responsible for Violations of Customary International Law: The U.S. Bombardments of Cambodia and Laos, Columbia Human Rights Law Review 32 (2001), S. 429 (437).

Für eine kritische Analyse der US-Politik insbesondere William Shawcross, Kissinger, Nixon and the Destruction of Cambodia (1980); für eine völkerrechtliche Bewertung auch Barrett (Anm. 1), S. $442 \mathrm{ff}$.

Die Literatur zur Herrschaft der Khmer Rouge ist umfangreich. Erwähnenswert sind insbesondere Elisabeth Becker, Wen the War was over. Cambodia and the Khmer Rouge (1986); Graig Etcheson, The Rise and Demise of Democratic Kampuchea (1984); Karl D. Jackson (Hg.), Cambodia 1975-1978. Rendezvous with Death (1989); Ben Kiernan, The Pol Pot Regime. Race, Power, and Genocide in Cambodia under the Khmer Rouge Revolution, 1975-1979 (1996); Michael Vickery, Cambodia 1975-1982 (1999); für weiter gespannte historische Darstellungen zu Kambodscha insbesondere John Tully, A Short History of Cambodia (2006); David Chandler, A History of Cambodia, 2. Auflage (1996); (speziell zur jüngeren Geschichte) ders., The Tragedy of Cambodian History (1991).

Für Biographien Pol Pot's s. David Chandler, Brother Number One. A Political Biography of Pol Pot (1999); Philip Short, Pol Pot. The History of a Nightmare (2004). Vgl. auch Karl D. Jackson, „Intellectual Origins of the Khmer Rouge”, in: ders. (Anm. 3), S. 241 ff. sowie Kenneth Quinn, „Explaining the Terror“, ebda., S. $213 \mathrm{ff}$. spektive s. neuerdings insbesondere Alexander Laban Hinton, Why did they kill? Cambodia in the Shadow of Genocide (2005). 
heiraten, kollektive Mahlzeiten, weitestgehende Abschottung von der Außenwelt, Abschaffung von Geld sowie die kompromisslose physische Vernichtung echter und vermeintlicher Regimegegner. Kambodscha war in diesen vier Jahren de facto ein riesiges Zwangsarbeitslager: über $95 \%$ der Bevölkerung lebten und arbeiteten in kollektiven Farmbetrieben. Strukturen von Recht, und seien es nur solche der gerechtigkeitsentleerten Art, der sich Diktaturen typischerweise bedienen, existierten in diesem System nicht ${ }^{6}$. Berichte über „Democratic Kampuchea“, wie sich dieser hermetisch abgeschlossene „Staat“ nannte, wurden in der Zeit seiner Existenz zunächst häufig für abwegig gehalten. Weder die reale Geschichte noch literarische Totalitarismusvisionen Orwell'schen Gepräges bildeten eine hinreichende Schreckensvorlage für dieses System. Im Gegensatz zu kommunistischen Diktaturen im Stile von Stalin, Tito oder Kim Il Sung gab es auch keinen Führerkult. Die Identität von Pol Pot blieb lange im Verborgenen. „Angkar“ (übersetzt schlicht: „,die Organisation“) war das omnipräsente und unheimlich entpersonalisierte Herrschaftsorgan der Khmer Rouge?

In den knapp vier Jahren der Herrschaft der Roten Khmer über das gesamte Kambodscha kamen wohl zwischen 1,5 und 2,5 Millionen Menschen systembedingt ums Leben ${ }^{8}$. Der wahrscheinlich überwiegende Teil der Opfer starb an Entkräftung, Auszehrung und nicht behandelten Krankheiten. Auf so genannten ,killing fields“ ${ }^{9}$, verstreut im ganzen Land, wurden aber auch hunderttausende Tötungsopfer vergraben. Im zentralen Verhör- und Foltergefängnis Tuol Sleng (,S 21“) in Phnom Penh, in das insbesondere die zahlreichen Opfer der innerparteilichen Säuberungsaktionen eingeliefert wurden, ,verhörte“ man ca. 17.000 Häftlinge, um sie nach Folter und Erzwingung aller denkbarer und undenkbarer Geständnisse manchmal vor Ort, zumeist aber erst auf dem außerhalb der Stadt gelegenen

Der einzige formelle Gesetzestext, der verabschiedet wurde, scheint die „Verfassung“ vom 5. Januar $1976 \mathrm{zu}$ sein (abgedruckt in: Raoul M. Jennar, The Cambodian Constitutions [19531993], 1995, S. 81 ff.). Dieser rudimentäre Text mit 21 knappen Artikeln blieb im Inland weithin unbekannt und richtete sich wohl auch intentional nur ans Ausland. Vgl. auch David Chandler, The Constitution of Democratic Kampuchea, Pacific Affairs 79 (1976), S. 505 ff.

Die Tatsache der Identität von Angkar mit der Communist Party of Kampuchea sowie die Führerschaft von Pol Pot wurden erst am 27. September 1977 öffentlich gemacht, möglicherweise nur auf chinesischen Druck hin (vgl. Becker [Anm. 3], S. 313). Dass in der Schlussphase der Herrschaft der Khmer Rouge ein Personenkult im Anmarsch war, ist freilich dadurch dokumentiert, dass einige Häftlinge in Tuol Sleng über Monate mit der Herstellung von Pol-Pot-Porträtgemälden und -Skulpturen beschäftigt waren (vgl. Vann Nath, A Cambodian Prison Portrait. One Year in the Khmer Rouge's S-21 [1998], S. 48 ff.).

Die konkreten Zahlen sind Gegenstand erheblicher Kontroversen, s. jüngst Ben Kiernan, The Demography of Genocide in Southeast Asia. The Death Tolls in Cambodia, 1975-79, and East Timor, 1975-80, Critical Asian Studies 35 (2003), S. 585 ff.; Craig Etcheson, After the Killing Fields. Lessons from the Cambodian Genocide (2005), S. $107 \mathrm{ff}$. 
„killing field“ Choeung Ek zu töten ${ }^{10}$. Es gab strikte Weisung, dass alle eingelieferten Personen zu töten seien. Nur sieben der eingelieferten Personen haben, soweit bekannt, die Einlieferung überlebt ${ }^{11}$. Mit Blick auf die zahlreichen Opfer innerparteilicher Säuberungen lässt sich sagen, dass wohl kaum eine Revolution, um das bekannte Wort Wolfgang Leonhardts zu benutzen, in so kurzer Zeit einen so hohen Prozentsatz ihrer eigenen Kinder gefressen hat. Gerichtsverfahren oder ähnliches gab es in „Democratic Kampuchea“ nicht, nicht einmal „Schauprozesse“ nach stalinistischem Muster. Etwas Bürokratismus gab es aber doch. In Tuol Sleng wurden alle eingehenden Opfer fotografiert, es wurden Verhörprotokolle angefertigt und spezielle Berichte geben Auskunft über die angewandten Verhörmethoden, zum Teil wurde sogar der Tod noch einmal fotografisch dokumentiert ${ }^{12}$. Spezifische Gewalt gegen Frauen scheint als gewissermaßen unerwünschter Begleiteffekt einer auf weitestgehende sexuelle Abstinenz der gesamten Bevölkerung zielenden Politik der Khmer Regime ebenfalls weit verbreitet gewesen zu sein, auch wenn hier bislang die Beweislage noch unsicher ist ${ }^{13}$. Historiker streiten sich, wie geplant die Verbrechen des Regimes in ihrer Gesamtheit waren, in jüngerer Zeit haben sich aber im Zuge immer besserer Aufarbeitung historischer Dokumente die Beweise dafür verdichtet, dass und wie die Führungsspitzen um Pol Pot und Nuon Chea involviert waren. Den Staatspräsidenten dieses Systems, der einstweilen in Freiheit nahe der thailändischen Grenze in Pailin lebt, hält freilich auch heute nichts davon ab, mit Verve publizistisch sein eigenes Unwissen hinsichtlich der Greueltaten zu behaupten ${ }^{14}$.

Choeung Ek ist heute eine zentrale Gedenkstätte. In einer großen Stupa sind hinter Glas über 8.000 Schädel aus den Gräbern nach Alter und Geschlecht sortiert aufgereiht; umliegend sind die freigelegten Massengräber zu besichtigen; der gesamte Boden ist nach wie vor durchsetzt mit Kleidungsfetzen. Die Regierung hat Anfang 2005 die Gedenkstätte, die gewissermaßen touristisches Pflichtprogramm in Phnom Penh ist, an ein japanisches Unternehmen zur Bewirtschaftung verpachtet, was bei lokalen NGOs einigen Protest auslöste.

Für den Erfahrungsbericht eines der wenigen Überlebenden s. Vann Nath (Anm. 7).

David Chandler, Voices from S-21: Terror and History in Pol Pot's Secret Prison (1999); ders, S21, the Wheel of History, and the Pathology of Terror in Democratic Kampuchea, in: Judy Ledgerwood (Hg.), Cambodia emerges from the Past: Eight Essays (2002), S. $16 \mathrm{ff}$.

Vgl. Katrina Anderson, Turning Reconciliation on its Head: Responding to Sexual Violence Under the Khmer Rouge, Seattle Journal for Social Justice 3 (2005), S. 785 (789 ff.); Kalyanee E. Mam. Democratic Kampuchea (1975-1979): Women as Instruments for Social Change, Phnom Penh 2000. Konsequenz der Anti-Sexualitäts-Politik der Khmer Rouge-Führung war nach den Ergebnissen der letztgenannten Untersuchung, dass Vergewaltigungen besonders häufig mit der Tötung der Opfer endeten, um keine Beweise zu hinterlassen (ebda, S. 26 ff.). 


\section{Politik der, ,Vergangenheitsbewältigung“}

Extremes Unrecht kann wohl nirgends einfach vergessen werden, aber der Umgang mit ihm ist ein erhebliches Problem. Unübersehbar ist, dass sich seit der Mitte des 20. Jahrhunderts und verschärft seit Beginn der 1990er Jahre die internationalen Standards für den Umgang mit der Vergangenheit verändert haben. Diese Entwicklung betrifft auch Kambodscha und das Bemühen, die Zeit der Khmer Rouge zu „bewältigen“.

Der Anfang jeder Verbrechensbewältigung liegt aber naturgemäß in der Beendigung des Unrechts selbst und der Art und Weise, wie diese Beendigung bewertet wird, so dass ein Blick zurück erforderlich ist. Insofern ist zunächst festzuhalten, dass man die Khmer Rouge von Seiten der Staatenwelt bis zum Ende ihrer Herrschaftszeit weithin ohne Belästigung agieren ließ. Obwohl schon vor ihrer Machtübernahme in Phnom Penh Berichte über die beispiellose Brutalität der Bewegung existierten und solche Berichte auch trotz der weitgehenden Abgeschlossenheit des Regimes vielfach durch Flüchtlinge bestätigt wurden, gab es international kaum eine Reaktion ${ }^{15}$. Als Vietnam, mit dem sich die Khmer Rouge auch noch in eine militärische Konfrontation verwickelt hatten, deren Herrschaft in Phnom Penh mit einer Invasion im Januar 1979 ein schnelles Ende machten, wurde, dessen Vorgehen im „Westen“, in China, Thailand und anderen Staaten der Region als Völkerrechtsbruch verdammt, obwohl es sich jedenfalls objektiv einigermaßen ersichtlich um eine rechtfertigungsfähige humanitäre Intervention handeln konnte ${ }^{16}$. Im Jahr 1979 herrschte allerdings Kalter Krieg und die simple Logik, nach der der Feind des Feindes ein Freund ist. Kambodscha blieb einstweilen, was es schon vor Machtübernahme der Khmer Rouge gewesen war: Spielball in den größeren regionalen und globalen Auseinandersetzungen ${ }^{17}$. Zwar gab es quer durch die 1980er Jahre und verstärkt seit deren Ende gerade auch in den USA immer wieder publizierte Überlegungen zur Notwendigkeit einer Verfolgung der kambod-

Für einen aufsehenerregenden frühen Bericht siehe insbesondere Francois Ponchaud, Cambodia Year Zero (1978, frz. Original: Cambodia Année Zéro, 1977); zur vielfach ungläubigen Reaktion und den ideologisch aufgeladenen Debatten in den USA etwa William Shawcross, The Quality of Mercy. Cambodia, Holocaust and Modern Conscience, (1984), S. 45 ff.

Für eine ausführliche völkerrechtliche Analyse, zugunsten der Völkerrechtskonformität der vietnamesischen Invasion argumentierend, Gary Klintworth, Vietnam's Intervention in Cambodia and International Law (1989); Christian Tomuschat, International Law. Ensuring the Survival of Mankind in the Eve of a new Century, RdC 291 (2001), S. 219, weist darauf hin, dass sich Vietnam offiziell auf das Recht auf Selbstvertreidigung und nicht auf menschenrechtliche Erwägungen stützte; aufgrund ,anderer Gründe“ Vietnams kritisch zur Annahme einer rechtfertigungsfähigen humanitären Intervention wohl auch Christian Hillgruber, in: Menzel/Pierling/Hoffmann, Völkerrechtsprechung (2005), Nr. 150 (S. 822 in Fn. 36); vgl. auch bereits Bazyler, Reexaming the Doctrine of Humanitarian Intervention in Light of the Atrocities in Kampuchea and Ethiopia, Stanford Journal of International Law 1987, S. 547 ff. $99 \mathrm{ff}$. 
schanischen Regierungsverbrechen zwischen 1975 und 1979 und auch die Einschaltung des Internationalen Gerichtshofs wurde vorgeschlagen ${ }^{18}$. Auf dem politischen Parkett spielten solche Ideen aber kaum eine Rolle. Dort wurde die Vertreibung der Khmer Rouge aus der Macht wie erwähnt als völkerrechtswidriger Akt Vietnams qualifiziert mit der Folge, dass den Khmer Rouge, nach einiger Zeit eingebunden in eine nur in der Gegnerschaft zu der Vietnam treuen Regierung verbundenen Koalition, in den Vereinten Nationen mit den Stimmen der meisten ,westlichen“ Staaten einschließlich der Bundesrepublik Deutschland das Recht zur Vertretung Kambodschas erhalten blieb ${ }^{19}$. Im Bestreben, Vietnam zu verdammen, ließ man sich sogar mit den Vertretern des aus der Herrschaft getriebenen Verbrecherregimes ein. Die Roten Khmer und ihre Koalitionäre operierten mit direkter Unterstützung Chinas und Thailands sowie nur notdürftig verschleiert der USA und anderer Staaten gegen die vietnamesisch und sowjetisch ,gesicherte“ Regierung in Phnom Penh ${ }^{20}$. Die in den Flüchtlingslagern im thailändischen Grenzgebiet zahlreich lebenden geflohenen Khmer Rouge erhielten auch von den Vereinten Nationen ein Vielfaches der Hilfe, die Kambodscha selbst zuteil wurde ${ }^{21}$ und im Übrigen wurde den Khmer Rouge weithin erlaubt, in diesen Lagern neu zu rekrutieren. An die Verfolgung vergangener Taten wurde international wenig gedacht. Westlichen Diplomaten war es zwar zum Teil von ihren Heimatregierungen verboten, die Vertreter der Khmer Rouge in New York auf dem Parkett der Vereinten Nationen mit Handschlag zu begrüßen, ihre dortige Präsenz blieb von solcher „Unfreundlichkeit“ freilich unberührt.

In Phnom Penh wurde 1979 eine pro-vietnamesische neue Regierung installiert ${ }^{22}$, während die geflohenen Roten Khmer von Rückzugspositionen aus weiter kämpften. Das Volk bewältigte die Vergangenheit zunächst im Wege zahlreicher ,informeller“ Revenge-killings gegenüber Roten Khmer und ihren Helfern. Genauere Daten hierüber sind aber soweit ersichtlich nicht vorhanden. Auf formeller Ebene wurde im August 1979 ein Strafverfahren in absentia gegen Diktator Pol Pot sowie seinen Außenminister Ieng Sary durchgeführt, zu

Insbesondere Gregory H. Stanton, The Cambodian Genocide and International Law, in: Ben Kiernan (ed.), Genocide and Democracy in Cambodia (1993).

19

Vgl. hierzu die kritische Darstellung bei Tom Fawthrop / Helen Jarvis, Getting Away with Genocide? Elusive Justice and the Khmer Rouge Tribunal (2004), S. 24 ff. (zum Abstimmungsverhalten der westlichen Staaten ebda. S. 33).

Vgl. Fawthrop/Jarvis (Anm. 19), S. 52 ff. Bekanntlich sprach Ronald Reagan in Formulierung der sog. Reagan Doktrin offen von der Unterstützung der „Freedom-Fighters“ in Kambodscha und Großbritannien gab jedenfalls im Nachhinein zu, den ,Widerstand“ militärisch trainiert zu haben.

Vgl. aus humanitärer Perspektive auch die Oxfam-Publikation von Eva Mysliwiec, Punishing the poor: the international isolation of Kampuchea (1988).

22

Maßgebliche Politiker der neuen Administration in Kambodscha einschließlich des amtierenden Premierministers waren dabei selbst bemerkenswerterweise ehemalige Rote Khmer, die sich während deren Herrschaft, angewidert von der Brutalität des Systems und vielleicht auch in der Furcht, selbst bald Opfer von Säuberungsaktionen zu werden, nach Vietnam abgesetzt hatten. 
dem auch ausländische Juristen eingeladen wurden ${ }^{23}$. Das üblicherweise als Schauprozess qualifizierte Verfahren, in dem freilich einiges an Beweisen aufgezeigt wurde, endete mit Todesurteilen, die gegenüber den geflohenen Verurteilten allerdings nicht vollstreckt werden konnten $^{24}$. In die Breite der Kader der Khmer Rouge wurde indessen nicht ermittelt, auch wenn es offenbar zum Teil insbesondere unmittelbar nach Machtübernahme und auch zur Verhängung zeitiger Inhaftierungen $\mathrm{kam}^{25}$. Hinsichtlich der Haupttäter wären ordnungsgemäße Verfahren mangels Zugriffs üblicherweise auch gar nicht möglich gewesen. Im Übrigen bestand die Vergangenheitspolitik der neuen Regierung darin, dass Regime um Pol Pot auf die Seite faschistischer Bewegungen zu stellen und damit in maximale Distanz zu sozialistischen Regierungsformen zu bringen. Man erklärte den 20. Mai zum „National Anger Day“ (gelegentlich zuspitzend als „National Hate Day“ ins Englische übersetzt), zu dem jährlich die Greuel der Khmer Rouge in Erinnerung gerufen werden sollten $^{26}$. Unmittelbar wurden Gedenkstätten eingerichtet, u.a. das Tuol-Sleng-Museum in Phnom Penh sowie das nahe der Hauptstadt gelegene „killing field“ Choueng Ek.

Als es seit dem Ende der 1980er Jahre zu internationalen Verhandlungen zur Beendigung des Bürgerkriegs kam, stellte nicht einmal die Reintegration der Khmer Rouge in den politischen Prozess ein Tabu dar. Vielmehr wurden sie zur Vertragspartei der abschließenden „Paris Agreements“, dem internationalen Vertrag, der das Mandat der Vereinten Nationen für eine übergangsweise Hoheit in Kambodscha begründete ${ }^{27}$ und damit die Grundlage für die „United Nations Transitional Authority in Cambodia“ (UNTAC) darstellte ${ }^{28}$. Erst nach

Vgl. die interessante Dokumentation der Prozessdokumente bei Howard J. De Nike, John Quigley and Kenneth J. Robinson, Genocide in Cambodia - Documents from the Trial of Pol Pot and Ieng Sary (2000). Das abschließende Urteil wurde auf Antrag Vietnams hin auch als UN-Dokument zirkuliert (UN-Doc. A/34/291 v. 20.9.1979). Vgl. zu dem Verfahren auch Fawthrop/Jarvis (Anm. 19), S. 40 ff.; Evan Gottesman, Cambodia After the Khmer Rouge. Inside the Politics of Nation Building (2002), S. 60 ff.; differenzierte Bewertung des Verfahrens z.B. bei Margaret Slocomb, The People's Republic of Kampuchea 1979-1989 (2003), S. 185.

Pol Pot starb 1998 nach offizieller Deutung ,natürlich“ aufgrund Herzversagens (dass er tot ist, scheint gewiss; hinsichtlich der Todesursache Restzweifel, vgl. dazu Fawthrop/Jarvis, [Anm. 19], S. 122 f.), nachdem er von den verbliebenen Khmer Rouge selbst in Haft genommen worden war. Ieng Sary wurde im Jahr 1996 im Gegenzug dafür, dass er sich von den Khmer Rouge lossagte, amnestiert (auf die Problematik dieser Amnestie mit Blick auf das Khmer Rouge Tribunal wird zurückzukommen sein). Er lebt aktuell komfortabel in Phnom Penh.

Gottesman (Anm. 23), S. 61 f.; entsprechender Bericht z.B. auch bei Huy Vannak, The Khmer Rouge Division 703. From Victory to Self Destruction (2003), S. 125.

Vgl. Fawthrop/Jarvis (Anm. 19), S. 74.

27 Abgedruckt in ILM 31 (1992), S. 180 ff.; eingehend Steven Ratner, The Cambodia Settlement Agreements, AJIL 87 (1993), S. 1 ff. 
dem innenpolitischen Zerbrechen dieser Politik der Wiedereinbindung wurden auch im Ausland die Stimmen relevanter, die eine Aufarbeitung der Khmer Rouge Verbrechen forderten. In den USA hatte sich schon in den späten 1980er Jahren eine Koalition von Nichtregierungsorganisationen unter dem Namen „Campaign to oppose the Return of the Khmer Rouge“ gegründet, deren Ziel darin lag, auf einen Richtungswechsel in der USamerikanischen Kambodscha-Politik hinzuwirken. Diese Bewegung feierte ihren zentralen Erfolg, als ein von ihr vorbereiteter Gesetzentwurf im Jahr 1994 als „Cambodian Genocide Justice Act“ erlassen wurde ${ }^{29}$. Das Gesetz stellte sodann die Grundlage für diplomatische Initiativen, aber auch für die Förderung groß angelegter Forschungsprogramme zur Untersuchung der Tatsachen sowie Rechtsfragen der Khmer-Rouge-Verbrechen dar. Im Kambodscha selbst unterlagen die Khmer Rouge derweil einem schrittweisen Marginalisierungsprozess. Pol Pot zog in den von den Khmer Rouge beherrschten Gebieten innerparteilich die Zügel wieder an mit dem Resultat, dass erhebliche Teile der Gefolgschaft wegbrachen. Nachdem 1996 der Außenminister der Khmer-Rouge-Zeit Ieng Sary dem Bürgerkrieg abgeschworen hatte und mit ihm fast die Hälfte der Truppen der Khmer Rouge übergelaufen war (um in die Royal Army eingegliedert zu werden), spitzte sich der Krieg innerhalb der Führungsspitze zu und kulminierte mit der von Pol Pot angeordneten Ermordung des langjährigen Weggefährten Son Senn und seiner Familie (einschließlich Kindern). Die potentiell nächsten Opfer innerparteilicher Säuberung um Ta Mok ergriffen die Initiative. Sie inhaftierten Pol Pot und machten ihm einen weiteren Schauprozess, in dem es freilich nicht um die Verbrechen seiner Herrschaftszeit 1975 bis 1979, sondern nur um die jüngsten innerparteilichen Säuberungsaktionen ging. Das von dem eingeladenen US-Journalisten Nate Thayer beobachtete Verfahren fand am 25. Juli 1997 statt und endete mit der Exekution von drei Gefolgsleuten sowie der Verurteilung Pol Pot's zu lebenslangem Hausarrest. Pol Pot starb neun Monate später nach offizieller Lesart im Schlaf an Herzversagen $^{30}$.

Der Zug zu einer vernünftigen gerichtlichen Aufarbeitung machte sich nur einige Tage vor dem Khmer-Rouge-internen „Verfahren“ gegen Pol Pot auf den langen Weg, als die kambodschanische Regierung in Gestalt der beiden Premierminister Ranariddh und Hun Sen eine Anregung der UN-Menschenrechtskommission ${ }^{31}$ aufgriffen und in einem Brief den UN-Generalsekretär um Unterstützung bei der Verfolgung der Verbrechen der Khmer

Serge Thion, Watching Cambodia (1993), der sarkastisch von der "United Nations Traditional Apathy in Cambodia" spricht (S. 186).

PL 103-236, 108 Stat. 486; 22 USC 2545 (1994).

30

Einige angesichts der Umstände kaum als ,,abenteuerlich“ zu bezeichnende Spekulationen gibt es freilich bis heute über die Möglichkeit von Selbstmord oder Mord.

31

Commission on Human Rights Resolution 1997/49, U.N. ESCOR, "Situation of Human Rights in Cambodia”, U.N. Doc. E/CN.4/RES/1997/49 (1997). 
Rouge baten ${ }^{32}$. Freilich blieb der Zug, um im Bild zu bleiben, schon kurz nach Abfahrt erstmals auf der Strecke liegen, als die Konflikte zwischen Hun Sen und Ranariddh militärisch eskalierten ${ }^{33}$ mit der Konsequenz der Flucht von Ranariddh und einer einjährigen internationalen Ächtung Kambodschas. Zum Ende des Jahres 1998 war die Situation sodann grundlegend verändert. Hun Sen war in journalistisch häufig kritisierten, von offiziellen Wahlbeobachtern aber im Wesentlichen akzeptierten Wahlen als einziger Premierminister bestätigt worden, Pol Pot war gestorben und die Khmer Rouge waren im Wesentlichen Geschichte. Ta Mok, der 1997 die Revolte gegen Pol Pot angeführt hatte und nachfolgend der einzige ,prominente“ Khmer Rouge Führer war, der nicht überlief, wurde 1999 verhaftet. „Duch“, der ehemalige Leiter des Foltergefängnisses Tuol Sleng, stellte sich einige Zeit später und ist seither ebenfalls in Haft. Ansonsten hat sich die kambodschanische Justiz soweit ersichtlich bislang fast nicht bemüht, Verbrechen der Khmer Rouge zu ermitteln oder zu bestrafen ${ }^{34}$. Stattdessen hat die Regierung auf ein anstehendes Tribunal verwiesen und in Vorbereitung hierauf letztlich auch die internationalen Verhandlungen mit den Vereinten Nationen nicht abbrechen lassen. Sie widersprach nun allerdings entschieden den Vorstellungen der Vereinten Nationen, dass ein internationales Gericht etabliert werden solle. Der UN-Generalsekretär hatte 1998 eine dreiköpfige „Group of Experts“ mit dem Auftrag eingesetzt, die Situation sowie die Optionen für ein Verfahren gegen die Khmer Rouge zu evaluieren. Der abschließende Bericht der Kommission, die sich für etwa eine Woche in Kambodscha selbst informiert hatte, plädierte strikt für ein Ad-

Brief an den Generalsekretär der Vereinten Nationen vom 21. Juni 1997, U.N. Doc. A/1997/488. Die historische Entwicklung der Verhandlungsprozesse ist bereits öfter beschrieben worden, vgl. etwa David Boyle, Establishing the Responsibility of the Khmer Rouge Leadership for International Crimes (2003, unveröffentlichtes Manuskript); Steve Heder, Hun Sen and Genocide Trials in Cambodia: International Impacts, Impunity, and Justice, in: Judy Ledgerwood (Hg.), Cambodia Emerges from the Past: Eight Essays (2002), S. 176 ff.; Stephen Heder / Brian Tittemore, Seven Candidates for Prosecution. Accountability for the Crimes of the Khmer Rouge, (2. Auflage 2001), S. 11 ff.; Daniel Kemper Donovan, Joint U.N.-Cambodia Efforts to Establish a Khmer Rouge Tribunal, Harvard International Law Journal 44 (2003), S. 551 ff.; Craig Etcheson, The Politics fo Genocide Justice in Cambodia, in: Cesare P.R. Romano / André Nollkaemper / Jann K. Klemper (Hg.), International Criminal Courts. Sierra Leone, East Timor, Kosovo, and Cambodia (2004), S. 181 ff.; geringfügig verändert wiederabgedruckt in: ders., After the Killing Fields. Lessons from the Cambodian Genocide, 2005, S. 141 ff.; ders. The A "Fair and Public Trial": A Political History of the Extraordinary Chambers, in: Justice Initiatives, A Publication of the Open Society Justice Initiative, Spring 2006 (abrufbar unter http://www.justiceinitiative.org), S. 7 ff.; Kelly Whitley, History of the Khmer Rouge Tribunal: Origins, Negotiations and Establishment, in: John D. Ciorciari (Hg.), The Khmer Rouge Tribunal (2006), S. 29 ff.

Bemerkenswerterweise eskalierte deren Konkurrenz speziell im Kontext des Versuchs von Ranarariddh's FUNCINPEC-Partei, die verbliebenen Khmer Rouge Kämpfer um Ta Mok in ihre Reihen zu integrieren und somit die eigene Machtposition gegenüber Hun Sen's CPP zu stärken. sacktouristen zu lebenslanger Haft verurteilt. Richter Sok Setha Mony, der das Urteil fällte, wurde kurze Zeit später 2003 von unbekannten Tätern ermordet. 
hoc-Gericht nach dem Muster der Gerichte für Jugsoslawien und Ruanda, das in einem asiatischen Staat außerhalb Kambodschas angesiedelt sein solle ${ }^{35}$. Angesichts des äußerst kritischen Grundtons, den der Bericht nicht nur mit Blick auf das kambodschanische Rechtssystem, sondern auch die kambodschanische Politik anschlug, konnte eigentlich kaum verwundern, dass er von Seiten der kambodschanischen Regierung entschieden zurückgewiesen wurde. Diese stellte sich fortan auf den Standpunkt, dass es sich bei dem Tribunal um ein zwar international unterstütztes, aber doch um ein nationales Verfahren im Rahmen des nationalen Rechts zu handeln habe ${ }^{36}$. Die Kompromisssuche gestaltete sich schwierig. Den ersten Durchbruch in Richtung zu einem Kompromiss erzielte im Oktober 1999 US-Senator John Kerry, der das Grundkonzept eines Vertrages zwischen den Vereinten Nationen mit sich anschließender nationaler Gesetzgebung sowie das Modell einer kambodschanischen Richtermehrheit in Kombination mit einer die internationale Seite stärkenden ,,super majority“ als Abstimmungsmodell vorschlug ${ }^{37}$. Aber auch nachdem auf dieser Grundlage 2001 national ein erstes Gesetz erlassen worden war, blieb die Situation gespannt. Zwischenzeitlich schien das Projekt gescheitert, als UN-Generalsekretär Kofi Annan 2002 den Abbruch der Verhandlungen verkünden ließ. Auch Institutionen wie Amnesty Internatonal äußerten scharfe Kritik an dem kambodschanischen Konzept ${ }^{38}$. Auf Druck verschiedener Staaten, aus dem Bereich der Vereinten Nationen selbst ${ }^{39}$ und schließlich einer Resolution der Generalversammlung der Vereinten Nationen ${ }^{40}$ wurden die Gespräche gleichwohl noch im gleichen Jahr offiziell wieder aufgenommen und im März 2003 kam es für alle Beobachter überraschend zu einem in Vertragsform fixierten Ergebnis ${ }^{41}$.

Report of the Group of Experts for Cambodia, UN Doc A/53/850-S/1999/231. Zum Bericht auch Steven R. Ratner, The United Nations Group of Experts for Cambodia, AJIL 93 (1999), S. 948 ff. Kommissionsmitglieder waren der der frühere Gouverneur Australiens Ninian Stephen, der frühere Chief Justice in Mauritius Rajsoomer Lallah sowie der US-amerikanische Professor Steven R. Ratner.

So schon Premierminister Hun Sen in einer Diskussion mit der UN-Group of Experts, vgl. Associated Press, 3 December 1998.

Vgl. A Marcher, The American Role in putting together a KR trial deal, Phnom Penh Post v. 28.4.2000.

Amnesty International, Cambodia: Cambodians Deserve International Standards of Justice (19. November 2002); dagegen aber mit guten Gründen Gregory H. Stanton, Perfection is the Enemy of Justice. A Response to Amnesty International's Critique of the Draft Agreement Between the U.N. and Cambodia, Bangkok Post 2003.

Staatlicherseits kam Kritik etwa von den USA, Australien, Japan, Frankreich, Russland und Indien, innerhalb der UN vom Menschenrechtsbeauftragten für Kambodscha Peter Leuprecht.

40 Resolution vom 18.12.2002, UN Doc. A/RES/57/228 (2002).

41

Agreement between the United Nations and the Royal Government of Cambodia concerning the prosecution under Cambodian Law of crimes committed during the period of Democratic Kampuchea, 17 March 2003, Report of the Secretary General on Khmer Rouge Trials, UN Doc. A/57/769, 31 March 2003; abrufbar auf der Internetseite der Government-Task Force zum Khmer 
Aufgrund von Problemen bei der Regierungsbildung, die sich in Kambodscha nach den Wahlen 2003 ergaben, verzögerte sich die Ratifizierung dieses Vertrages, die dann aber nach Ende dieser Krise im Juni 2004 ebenso erfolgte wie die Anpassung des nationalen Gesetzes über die „Extraordinary Chambers“, dessen Ursprungsversion bereits 2001 verabschiedet worden war ${ }^{42}$. Langsam aber doch kontinuierlich nehmen die Dinge seither ihren Lauf. Für etwas Diskussion sorgte die endgültige Bestimmung des Gerichtsgebäudes ${ }^{43}$. Als schwieriger erwies sich die Suche nach dem Geld für das Verfahren ${ }^{44}$ und umstritten war naturgemäß zuletzt die Richterauswahl. Inzwischen scheinen die Dinge aber organisatorisch gerichtet und nachdem am 3. Juli 2006 Richter und Staatsanwälte vom König vereidigt wurden, kann nun die eigentliche Arbeit beginnen. Just vor der Vereidigung machte freilich Ta Mok Schlagzeilen. Schwer krank wurde der inzwischen 82 jährige ehemalige Khmer Rouge Kader in ein Militärkrankenhaus eingeliefert; wo er am 22. Juli verstarb. Zwangsläufig erinnert der Tod eines der Hauptkandidaten für das Verfahren an die Frage, warum es seit 1996 noch einmal zehn Jahre gedauert hat, die Verfahren „ins Rollen“ zu bringen. Hierüber ist freilich bereits genügend argumentiert und geschrieben worden: Während die einen die Verantwortung bei der Regierung Kambodschas suchen, weisen andere auch (nicht zu Unrecht) auf Fehler und Ungeschicklichkeiten der Vereinten Nationen hin ${ }^{45}$.

Rouge Tribunal (www.cambodia.gov/krt/). Die Vereinbarung wurde von Kambodscha unmittelbar am 28. März, von der UN-Generalversammlung am 13. Mai 2003 akzeptiert.

Law on the Establishment of the Extraordinary Chambers, with inclusion of amendments as promulgated on 27 October 2004 (NSS/RKM/1004/006) (www.cambodia.gov/krt/).

Ursprünglich war das Chaktomuk-Theater inmitten Phnom Penhs vorgesehen, die Regierung entschied sich aber schließlich, einen neu errichteten Gebäudekomplex am Stadtrand für das Verfahren einzurichten. Von Seiten nationaler NGOs wurde kritisiert, dass es sich um eine für das Militär erbaute Einrichtung handelt, woraus die Möglichkeit der Einschüchterung von Zeugen etc. folge. Die Vereinten Nationen haben der Ortswahl jedoch zugestimmt und in der Tat wird man konstatieren können, dass die Räumlichkeiten insgesamt wohl geeigneter sind als das ursprünglich vorgesehene Theater (abgesehen davon, dass auch dieser Gerichtsort seinen Beigeschmack gehabt hätte, fand in ihm doch 1979 bereits der „Schauprozess“ gegen Pol Pot und Ieng Sary statt).

Der internationale Finanzierungsanteil des vorgesehenen Gesamtetats 56 Mio. Dollar wird etwa zur Hälfte von Japan getragen, das mit 21,6 Mio. Dollar den mit Abstand größten Beitrag leistet. Deutschland ist mit einem zugesagten Betrag von ca. 2,9 Mio. Dollar nach Frankreich (4,8 Mio. Dollar) derzeit der drittgrößte Beitragszahler (Namibia hat mit 500 Dollar den kleinsten Unterstützungsbeitrag zugesagt). Nachdem auf der internationalen Seite die Zusagen weitgehend beisammen waren, erklärte Kambodscha, von dem eigenen Beitrag in Höhe von ca. 13,5 Mio. Dollar nur 1,5 Mio. Dollar selbst tragen zu können. Zeitweilig schien die kambodschanische Regierung über die Finanzierung noch einmal auf Zeit zu spielen, schließlich sagten aber Indien und die Europäische Union Beiträge zu und eine Reihe von Staaten willigte in die Verwendung von Restmitteln in der Höhe von 5 Mio. Dollar eines UN-Treuhandfonds der UNTAC-Zeit für den kambodschanischen Anteil zu, so dass zur Zeit der Richtervereidigung Anfang Juli 2006 ,nur noch“ ein offener Restfinanzierungsbetrag von etwa 4 Mio. Dollar national und 2 Mio. Dollar international verblieb. 
Einigkeit bestand jedenfalls zuletzt, dass sich das Tribunal wohl entweder alsbald oder nie materialisieren würde.

\section{Die ,Extraordinary Chambers“}

Das nationale Gesetz über die „Extraordinary Chambers in the Courts of Cambodia“ (ECCC) wurde wie erwähnt zuerst 2001 erlassen. Es schien in seiner zunächst vom Parlament beschlossenen Version aufgrund des Verweises auf das kambodschanische Strafgesetzbuch von 1956 die Todesstrafe zuzulassen, was freilich unmittelbar vom Constitutional Council zutreffend als unvereinbar mit Art. 32 Abs. 2 der Verfassung (,,There shall be no capital punishment") qualifiziert ${ }^{46}$ und darauf hin korrigiert wurde. Das Gesetz wurde sodann im Oktober 2004 novelliert, um den Maßgaben der zwischenzeitlich erzielten Vereinbarung mit den Vereinten Nationen zu entsprechen ${ }^{47}$. Es sieht nunmehr in zwei Instanzen (nach der Version 2001 hätte es noch drei Instanzen geben sollen) gemischte Kammern vor, die in der ersten Instanz aus drei kambodschanischen und zwei internationalen Richtern und in der zweiten Instanz aus vier kambodschanischen und drei internationalen Richtern bestehen. Der Präsident wird jeweils aus der Reihe der kambodschanischen Richter zu bestimmen sein. Alle Richter werden durch den kambodschanischen ,Supreme Council of Magistracy“ ausgewählt, wobei bezüglich der internationalen Richter aus einer vom Generalsekretär der Vereinten Nationen vorzulegenden Liste auszuwählen ist, die mindestens sieben Vorschläge zu umfassen hat. Während die internationale Seite also formal keinerlei Einfluss auf die Auswahl der kambodschanischen Richter hatte, konnte die kambodschanische Seite immerhin in einem gewissen Umfang über den Einsatz der internationalen Richter entscheiden ${ }^{48}$. Teil des Kompromisses zwischen der kambodschanischen Regierung

Rouge, Virginia Law Review 90 (2004), S. 893 ff.; andererseits Gerald V. May III, An (Un)Likely Culprit: Examing the U.N.'s Contraproductive Role in the Negotiations over a Khmer Rouge Tribunal, Boston College International and Comparative Law Review 27 (2004), S. 147 ff. Zu einer fairen Beurteilung gelangt man wohl nur, wenn man auf beiden Seiten nach Ursachen sucht, wobei es zum großen Teil auch weniger um Schuld gehen muss als um objektive Gründe, die den Verhandlungsprozess verkomplizierten. Während die UN in verschiedenen Zusammenhängen fast gleichzeitig verschiedene Modelle eines völlig neuartigen Konzepts gemischter Gerichte zu erproben hatten, bedeutet das Thema für die kambodschanische Regierung aus einer ganzen Palette an Gründen einen innen- und außenpolitischen Drahtseilakt.

Constitutional Council vom 12.2.2001, Case No. 038/001/2001.

47

$\mathrm{Zu}$ der durch die Konzeption des Gerichts vgl. neben den Beiträgen von Boyle, Donovan und Heder/Tittemore (Anm. 32), auch Ernestine E. Meijer, The Extraordinary Chambers in the Courts of Cambodia for Prosecuting Crimes Committed by the Khmer Rouge: Jurisdiction, Organization, and Procedure of an Internationalized National Tribunal, in: Romano (Anm. 32), S. 207 ff.; eine Reihe von Einzelaspekten wird diskutiert in Ciorciari (Anm. 32).

Sie konnte nicht nur zwei der sieben Richter zunächst außen vor lassen, sondern auch bestimmen, welche zwei der sieben Richter zunächst in erster Instanz tätig sind. Für die Ermittlungsrichter blieb ebenfalls Spielraum, weil der UN-Generalsekretär zwei Kandidaten zu benennen hatte, von 
und den Vereinten Nationen ist auch das Prinzip der so genannten ,super majority“, nach dem die Entscheidungen erstinstanzlich von vier, zweitinstanzlich von fünf Richtern getragen werden müssen, die internationalen Richter also nicht kollektiv überstimmt werden können. Die einzurichtenden Strafverfolgungsorgane werden aus einem kambodschanischen und einem internationalen Staatsanwalt sowie einem kambodschanischen und einem internationalen Ermittlungsrichter bestehen, die grundsätzlich einvernehmlich handeln sollen. Im Fall, dass zwischen nationaler und internationaler Seite unterschiedliche Auffassungen nicht ausgeräumt werden können, werden die betreffenden Entscheidungen einer speziellen Richterkammer (pre trial chamber) zur Entscheidung zugewiesen. Kann sich diese wiederum nicht einigen, gilt für Mehrheitsentscheidungen erneut die super-majority, freilich zugunsten investigationswilligen Seite, so dass die Ermittlungen voranschreiten können, soweit sich nicht eine entsprechende Mehrheit dagegen ausspricht.

Die ECCC haben Jurisdiktion für ,senior leaders“ des Khmer Rouge Regimes sowie diejenigen, die „most responsible“ für die Verbrechen dieses Regimes in der Zeit seiner Herrschaft in Kambodscha sind. Zeitlich ist der Rahmen präzise datiert und reicht vom 17. April 1975 bis zum 6. Januar 1979. Der Fokus des Gerichts ist damit in mehrfacher Hinsicht begrenzt. Es geht nur um Verbrechen innerhalb der formalen Herrschaftszeit der Khmer Rouge über den Staat als Ganzen, nicht um solche während des vorangehenden oder nachfolgenden Bürger- bzw. Guerillakrieges. Ausgeschlossen sind auch Verbrechen sonstiger Konfliktparteien und schließlich bleiben die Verfahren auf Hauptverantwortliche begrenzt. Die von den ECCC zu verfolgenden Straftaten ${ }^{49}$ sind im Gesetz definiert. Zwar gibt es einige Wortlaut-Divergenzen zwischen dem Gesetz, dem Agreement sowie den zum Teil in Bezug genommenen internationalen Konventionen (Genozid-Konvention, ICC-Statut), diese dürften sich aber im Wege der Auslegung bereinigen lassen. In Rede stehen die inzwischen klassischen Straftaten des Völkerstrafrechts (Genozid, Verbrechen gegen die Menschlichkeit, Kriegsverbrechen) sowie einige Straftaten unter dem Strafgesetzbuch des Königreichs Kambodschas aus dem Jahr 1956 (Homizid, Folter, religiöse Verfolgung), von dessen Fortgeltung in der Zeit der Khmer Rouge der Gesetzgeber offensichtlich ausgeht und für die eine ursprünglich geltende zehnjährige Verjährungsfrist nachträglich nun auf dreißig Jahre ausgedehnt worden ist ${ }^{50}$. Das Strafverfahrensrecht soll sich nach den Regeln

denen kambodschanischerseits zunächst einer ausgewählt werden konnte (Art. 5 Abs. 5 des Agreements). Gleiches galt für die Besetzung der internationalen Staatsanwaltsstelle (Art. 6 Abs. 5 des Agreements). (Anm. 32), S. 55 ff.; s. auch Meijer (Anm. 47), S. 212 ff. (noch auf der Grundlage der Gesetzesversion 2001). 
des kambodschanischen Rechts richten; wo dieses internationalen Maßstäben nicht genügend, könnten letztere ergänzend herangezogen werden. Einige Verfahrensgarantien sind im Gesetz auch ausdrücklich formuliert.

\section{Extraordinary Chambers und Völkerstrafrecht}

Die ECCC werden, so sie denn ihren Auftrag ausführen, in der Entwicklungslinie des modernen Völkerstrafrechts liegen, die bekanntlich im Wesentlichen mit den ,Nürnberger Prozessen" nach dem Ende des Zweiten Weltkriegs ihren Anfang nahm ${ }^{51}$, sich aber im Grunde erst seit dem Beginn der 1990er Jahre anschickt, etwas Nachhaltigkeit zu versprechen. In den 1950er bis 1980er Jahren war ein internationales Bemühen um die Verfolgung staatsinterner Verbrechen kaum zu verzeichnen. Brutale Diktatoren wie Haitis Duvalier oder Ugandas Idi Amin verlebten auch nach dem Ende ihrer Herrschaft üblicherweise einen komfortablen Ruhestand irgendwo im Exil. In den 1990er Jahren änderten sich die Rahmenbedingungen indessen. Nach dem Ende des Kalten Krieges, während dessen menschenrechtliche Erwägungen auf allen Seiten eher untergeordnet waren, veränderte sich die Situation indessen grundsätzlich. „Völkerstrafrecht“ hat sich im Sog einer dynamischen Entwicklung des Menschenrechtsgedankens ${ }^{52}$ innerhalb kürzester Zeit praktisch und theoretisch von der Unauffindbarkeit zur Unüberschaubarkeit entwickelt ${ }^{53}$. Die Einrichtung von Ad-hoc-Gerichten für Ruanda und Jugoslawien auf der Grundlage von Chapter VII der UN-Charta, die Etablierung eines Internationalen Strafgerichtshofs, die Bemühungen nationaler Jurisdiktionen, fremde Staatsverbrecher vor Gericht zu ziehen („Pinochet“) und schließlich auch die Einrichtung erster gemischter (,hybrider“) international-nationaler Gerichte sind Aspekte dieser Revolutionierung des Völkerrechts auf der institutionellen Ebene, zu der nun auch der späte Versuch einer Aufarbeitung der Khmer Rouge Verbrechen gehört.

Gerichte des Völkerstrafrechts sind die ECCC dabei schon deshalb, weil sie im Schwerpunkt (wenn auch nicht ausschließlich), ,international crimes“ zum Gegenstand haben.

ist (hierzu auch sogleich unter V.). Freilich lässt sich gerade mit Blick auf schwerste Staatsverbrechen auch im Völkerrecht die Tendenz feststellen, Verjährung nicht oder nur eingeschränkt zu ermöglichen. Im Übrigen hat auch in Deutschland der Gesetzgeber Verjährungsfristen im Angesicht drohender Verjährung nationalsozialistischer Straftaten verlängert (freilich vor Ablauf der ursprünglichen Fristen) und das Bundesverfassungsgericht hat hierzu festgestellt, dass das Rückwirkungsverbot des Art. 103 Abs. 2 GG auf Verjährungsbestimmungen keine Anwendung findet (BVerfGE 25, 269 [286 ff.]).

Retrospektiv zu den Nürnberger und Tokioter Prozessen zuletzt Jeannine Hoffmann, in: Menzel/ Pierlings/Hoffmann (Hg.) (Anm. 16), Nrn. 142, 143.

52

Gerhard Werle, Menschenrechtsschutz durch Völkerstrafrecht, ZStW 109 (1997), S. 808 ff.

53

Zur Entwicklung Gerhard Werle, Völkerstrafrecht (2003), Rn. 1 ff. mwN. 
Betrachtet man zunächst die in Rede stehenden Straftaten, sind wie in den übrigen zwischenzeitlich installierten internationalen bzw. semi-internationalen Spezialgerichten sowie dem Römischen Statut für den Internationalen Strafgerichtshof ${ }^{54}$ die Straftatbestände des Völkermordes (Genozids), der Verbrechen gegen die Menschlichkeit sowie Kriegsverbrechen $^{55}$. Zusätzlich aufgenommen sind, über den klassischen Katalog internationaler Verbrechen hinausgehend, Verstöße gegen Haager Konvention zum Schutz von Kulturgütern in bewaffneten Konflikten (Art. 7) sowie solche gegen diplomatisch geschützte Personen (Art. 8) ${ }^{56}$. Wenn im Übrigen, durchaus prominent vorangestellt, auch einige Straftatbestände des nationalen Rechts aufgenommen sind (Tötungsdelikte, Folter, religiöse Verfolgung) so bestätigt dies mit Blick auf die enge Auswahl dieser Tatbestände als Ausnahme die Regel, dass es im Schwerpunkt um ,internationale“ Verbrechen geht, schon weil auf dieses nationale Recht nicht allgemein, sondern nur höchst ausschnittsweise zugegriffen wird $^{57}$. Mit Blick auf Organisations- und Verfahrensrecht lassen sich die ECCC dem Völkerstrafrecht ebenfalls zurechnen, weil sie in Ausführung eines internationalen Vertrages eingerichtet werden, weil sie überwiegend international finanziert sind und schließlich, weil in ihnen (auch) ,internationale“ Richter tätig werden. Auch wenn die kambodschanische Seite betont, dass es sich bei den ECCC um nationale Justizeinrichtungen handeln wird, implizieren die genannten Faktoren einen Grad an „Internationalisierung“, der die ECCC doch zweifellos als in besonderer Weise ,internationalisiert“ erscheinen lässt.

Das Khmer Rouge Tribunal gehört mithin zur Gattung der „hybrid courts“, also gemischt national-internationaler Gerichte. Dieser Typus ist in Abkehr vom Modell der Internationalen Strafgerichtshöfe für Jugoslawien und Ruanda, bei denen es sich um rein internationale Gerichte handelt, erst in jüngster Zeit entwickelt worden, findet sich inzwischen aber schon in Ost-Timor, Sierra Leone sowie dem Kosovo ${ }^{58}$. Untereinander divergieren diese

Zur Einrichtung des „ICC“ statt aller Klaus-Ferdinand Gaerditz, in: Menzel/Pierlings/Hoffmann (Anm. 16), Nr. 5 mit zahlreichen Nachweisen.

Was bemerkenswerterweise fehlt, ist das „Nürnberger“ Verbrechen des Aggressionskrieges. Sein Fehlen vermeidet aber nicht nur möglicherweise schwierige emotionale Aufladungen, er liegt auch auf der Linie des ICC-Statuts, in dem das Aggressionsdelikt einstweilen auch suspendiert bleibt. Im Übrigen offenbart sich hier schlicht ein sachlicher Unterschied zwischen „Nürnberg“, das im Kern ein „Kriegsverbrecherprozess“ war, und „Phnom Penh“, wo es zentral um interne Gewalt gehen wird.

Vgl. Bert Swart, Internationalized Courts and Substantive International Law, in: Romano (Anm. 32), S. 291 (299 f.). Praktisch mögen diese Delikte, sollten sie von den Strafverfolgungsorganen aktualisiert werden, manche Schwierigkeiten aufwerfen, weil sie im Völkerstrafrecht nicht umfänglich etabliert und diskutiert sind.

Zu den Gründen der Einbeziehung nationaler Straftatbestände auch Swart (Anm. 56), S. 303 f. in International Criminal Justice: Kosovo, East Timor, Sierra Leone and Cambodia, 2003; übersichtlich Ilias Bantekas \& Susan Nash, International Criminal Law (2. Auflage 2003), S. 397 ff. 
gemischten Gerichte nicht unerheblich. Es handelt sich um kein dogmatisch konzipiertes Modell, sondern einstweilen eher um eine pragmatisch in unterschiedlichen Zusammenhängen entwickelte Erscheinung der völkerstrafrechtlichen Lebenswelt ${ }^{59}$. In Sierra Leone wurde auf Initiative des UN-Sicherheitsrates zwischen den Vereinten Nationen und der Regierung von Sierra Leone im Jahr 2000 die Einrichtung eines „Special Court“, dessen Rechtsgrundlage unmittelbar der Vertrag ist und dessen Richterkollegium in erster Instanz aus jeweils zwei internationalen Richtern und einem nationalen Richter sowie in zweiter Instanz aus drei nationalen und zwei internationalen Richtern besteht. In Ost-Timor, dessen Entwicklung in diesem Bereich in Kambodscha schon wegen der regionalen Nähe besonders genau beobachtet wird ${ }^{60}$, hat die UN-Übergangsverwaltung „UNTAET“ im Jahr 2000 kraft ihrer Gesetzgebungsgewalt spezielle Panels beim District Court eingerichtet, in dem jeweils zwei internationale Richter und ein nationaler Richter bzw. - in schweren Fällen drei internationale Richter und zwei nationale Richter das Entscheidungsgremium bilden. Im Kosovo scheiterten die Bemühungen, im Rahmen von „UNMIK“, ein echtes ,internationalisiertes" Gericht einzurichten, und daher beschränkt man sich dort darauf, sporadisch internationale Richter und Staatsanwälte den lokalen Gerichten zuzuordnen. Konzeptionell drängt sich auf, dass die ECCC am ehesten mit dem Modell in Sierra Leone zu vergleichen sind, denn hier wie dort basiert das Gericht jedenfalls auf dem Vertrag zwischen einem souveränen Staat und den Vereinten Nationen. Ersichtlich ist aber auch, dass die Verhandlungen über das Gericht in Sierra Leone weit weniger von Souveränitätsvorbehalten der nationalen Regierung geprägt waren, so dass ein Gericht entstand, das strukturell weit ,internationaler“ ist als die kambodschanischen $\mathrm{ECCC}^{61}$.

Trotz der erheblichen Unterschiede lassen sich die gemischten Gerichte gemeinsam dem Konzept vollständiger Internationalität gegenüberstellen. Vor- und Nachteile des Mischmodells liegen dabei auf der Hand. Für solche Gerichte sprechen ein größeres „ownership“ der nationalen Seite und damit verbunden mögliche Lerneffekte für die häufig desolaten nationalen Rechtsordnungen sowie nicht zuletzt Kostenvorteile. Negativ verbuchen bisweilen strukturelle und verfahrensrechtliche Schwierigkeiten. Akzeptanzprobleme können so oder so entstehen und hängen im Einzelnen von den konkreten Umständen ab. All dies ist in jüngerer Zeit intensiv diskutiert worden und hier ist nicht der Raum, diesen Fragen in übergreifender Allgemeinheit nachzugehen. Allgemein sei nur angemerkt, dass die Probleme der Gerichte in diesem Tätigkeitsbereich nicht notwendig Konsequenzen

Vgl. nur Daphna Shraga, The Second Generation UN-Based Tribunals: A Diversity of Mixed Jurisdictions, in: Romano (Anm. 32), S. 15 (36 ff.).

60

Eine literarische Vergleichsperspektive etwa bei Suzannah Linton, New Approaches to international justice in Cambodia and East Timor, IRRC 84 (2002), S. 93 ff. 
einer spezifischen rechtlichen Konstruktion sind, sondern mit der generell fundamentalen Schwierigkeit zu tun haben, extremes Staatsunrecht oder extreme Massenverbrechen der in Rede stehenden Art justiziell aufzuarbeiten. Der Blick auf „Nürnberg“, „Tokio“, „Den Haag“, „Arusha“ etc. erweist, dass noch kein solches Gericht ohne erhebliche Probleme geblieben ist. Nürnberg und Tokio waren „Siegertribunale“ und betraten zudem völkerrechtliches Neuland mit allen damit verbundenen Schwierigkeiten sub specie Rückwirkungsverbot etc.. Der rechtslogistische Perfektionismus der Yugoslawien und Ruanda betreffenden Gerichte (ICTY bzw. ICTR) generiert zwar Entscheidungen, die aufgrund ihrer Gründlichkeit mustergültig erscheinen, die aber schon angesichts ihrer typischen Umfänge häufig kaum mehr kommunizierbar sind und die im Übrigen unter einem nicht beliebig wiederholbaren politischen und logistischen Aufwand ermöglicht wurden. Hinsichtlich ihrer Erfolgsbilanzen sind auch diese Gerichte noch nicht im grünen Bereich. In Den Haag schien der Hauptangeklagte Milosovic bisweilen mit dem Gericht zu spielen und einige weitere Hauptkandidaten für Verfahren werden dem Gericht seit Jahren beharrlich nicht zugeführt. In Arusha (dem Sitz des Ruanda-Gerichtshofs) ist das Verhältnis zur ruandischen Regierung, die selbst Strafverfolgung unternommen hat, in einer Weise gespannt, die fast jede Zusammenarbeit auszuschließen scheint. Der nachhaltigste Effekt von ICTY und ICTR liegt vielleicht letztlich nicht in ihrer konkreten Erfolgsbilanz mit Blick auf eine Aburteilung der Verantwortlichen der jeweils in Rede stehenden Verbrechen, sondern darin, dass sie mit ihren fast unbegrenzt erscheinenden Milliardenbudgets als Begleiteffekt die Voraussetzungen verbessert haben, unter denen gemischte und nationale Gerichte heute arbeiten können. Sie haben eine erhebliche Zahl über das Internet gut zugänglicher Entscheidungen produziert, in denen das Völkerstrafrecht im Detail konkretisiert wird und sie haben als Beschäftungsstellen für zahlreiche Richter, Staatsanwälte, Rechtsanwälte etc. einen erheblichen Stamm an Völkerstrafrechtspraktikern und -experten erzeugt, die nun durch die Welt flotieren und auch gemischten oder gar rein nationalen Bemühungen um justizielle Vergangenheitsbewältigung zur Verfügung stehen. In Phnom Penh jedenfalls verging schon 2005 kaum eine Woche, in der nicht ein „Experte“ aus Den Haag, Arusha oder Dili seine Erfahrungen vermittelte. Im Kontext der Arbeit dieser Gerichte sowie der Einrichtung des Internationalen Strafgerichtshofs, dessen Einrichtung seinerseits über sie einen maßgeblichen Anschub erhielt, wurde im Übrigen in kürzester Zeit ein erhebliches $\mathrm{Ma}$ wissenschaftlicher Durchdringung erreicht, von der die ECCC ebenfalls profitieren können.

Die Bereitstellung eines Systems justizieller Aufarbeitung extremen Unrechts scheint zwar inzwischen weltweit zu den natürlichen Erwartungshaltungen der internationalen Gemeinschaft sowie der lokal betroffenen Gesellschaften zu gehören, Schwierigkeiten im Detail sind hiermit aber mitnichten unerheblich. Welches Gerichtsmodell am besten passt oder welches überhaupt realisierbar ist, hängt von verschiedenen Faktoren ab und komparative Analysen sollten nicht zu dem Fehlschluss verleiten, es ließe sich in jedem Fall rechtlich oder faktisch frei entscheiden, welcher Weg zu beschreiten ist. Hinsichtlich Kambodschas 
ist insoweit z.B. bezweifelt worden, dass die Einrichtung eines dritten Ad-hoc-Gerichts nach dem Muster der Gerichtshöfe für Jugoslawien und Ruanda bei Einhaltung juristischer Regeln überhaupt ein gangbarer Weg gewesen wäre. Bedenken resultieren insofern nicht nur pragmatisch aus einem bisweilen prognostizierten chinesischen Veto im Sicherheitsrat der Vereinten Nationen ${ }^{62}$ oder allgemeinen Erwägungen zu einer „tribunale fatigue“ des Sicherheitsrates $^{63}$, sondern auch daraus, dass Beschlüsse auf der Grundlage von Chapter VII der UN-Charta in juristischer Hinsicht eigentlich nicht beliebig getroffen werden können, sondern an gewisse tatbestandliche Voraussetzungen gebunden sind ${ }^{64}$. Aber es sind nicht nur, und wohl nicht einmal überwiegend rechtliche Gründe, die einen Trend zur „Nationalisierung“ solcher Verfahren fördern. Wohl aufgrund einer ganzen Reihe von Gründen scheint es sinnvoll, die Verfolgung auch fundamentaler Verbrechen im innerstaatlichen Bereich soweit möglich wieder in die Hände nationaler Jurisdiktionen zu legen ${ }^{65}$. Auch das Konzept des nunmehr eingerichteten Internationalen Strafgerichtshofs beruht in diesem Sinne auf einer nur residualen Zuständigkeit desselben. Die Konjunktur der gemischten Gerichte liegt jedenfalls auf der Linie, nationale Verantwortung zu betonen, aber internationale Sicherungsmechanismen vorzuhalten. Zwar ist hiermit noch nicht konkret entschieden, wie sich das Verhältnis zum Internationalen Strafgerichtshof ggf. gestaltet, es liegt aber nahe, dass jedenfalls ein Gericht wie dasjenige in Kambodscha als nationales Gericht im Sinne von Art. 17 des ICC-Statuts angesehen werden kann, der den Vorrang nationaler vor internationaler Jurisdiktion statuiert ${ }^{66}$, wobei es im konkreten Fall zu Konflikten mit der Jurisdiktion des ICC ohnehin nicht kommen kann, weil die in Rede stehenden Taten schon zeitlich nicht in dessen Zuständigkeit fallen ${ }^{67}$.

Vgl. Boyle (Anm. 32), S. 12.

Antonio Cassese, International Law, 2. Auflage 2005, S. 458; Steven R. Ratner / Jason S. Abrams, Accountability for Human Rights Atrocities in International Law. Beyond the Nuremberg Legacy (2. Auflage 2001), S. 318.

Vgl. Boyle (Anm. 32), S. 12. Für "more problematic" im Vergleich zu Jugoslawien und Ruanda halten den Weg über Chapter VII in Kambodscha auch Ratner/Abrams (Anm. 63), S. 317.

Umfassend William Burke-White, A Community of Courts: Toward a System of International Criminal Law Enforcement, Michigan Journal of International Law 24 (2002), S. 1 ff.

Markus Benzing / Morten Bergsmo, Some Tentative Remarks on the Relationship Between Internationalized Criminal Jurisdictions and the International Criminal Court, in: Romano (Anm. 32), S. 407 (412 f.) halten sogar Gerichte wie diejenigen in Kosovo und Sierra Leone (konzeptionell weit internationaler als dasjenige in Kambodscha) für unter Art. 17 subsumierbar.

Die Kompetenz des ICC ist nicht retroaktiv (Art. 24 ICC-Statut), kann also auch für Gründungsmitglied Kambodscha, das seine Ratifizierungsurkunde am 11. April 2002 in New York hinterlegte, nur Taten nach Inkrafttreten des Vertrages am 1. Juli 2002 erfassen. 
Die Zukunftsperspektiven des Konzepts gemischter Gerichte ${ }^{68}$ werden jedenfalls partiell davon abhängen, in welchem Umfang die erste Generation, zu der man das anstehende Khmer Rouge Tribunal noch wird rechnen können, die Leistungsfähigkeit des Konzepts erweist. Mit Blick auf Ost-Timor wird bislang durchaus kritisch zwischenbilanziert ${ }^{69}$, während für Sierra Leone einstweilen die positiven Eindrücke zu überwiegen scheinen ${ }^{70}$. In Kambodscha wurde das Gericht gelegentlich schon zur Farce erklärt, bevor es überhaupt eingerichtet ist ${ }^{71}$. Angesichts der Vorgeschichte stehen die Entwicklungen hier jedenfalls unter genauer Beobachtung. Gerade deshalb ist auch für die Entwicklung des Völkerstrafrechts interessant, vor welchen praktischen und rechtlichen Herausforderungen die ECCC in Kambodscha konkret stehen werden, wenn sie denn in naher Zukunft tatsächlich ihre Arbeit aufnehmen.

\section{Herausforderungen}

Die erste große Herausforderung lag sicher in der Einrichtung des Tribunals selbst, denn weder war das volle Engagement der kambodschanischen Regierung zu jeder Zeit zweifelsfrei, noch blieben Obstruktionsversuche aus dem Ausland aus ${ }^{72}$. Wenn also nun am 3. Juli 2006 Richter und Strafverfolger vereidigt wurden und sie jetzt schrittweise ihre Arbeit aufnehmen, ist die erste große Hürde bereits genommen. Viele weitere Schwierigkeiten werden folgen. $\mathrm{Ob}$ die kambodschanischen Richter und Strafverfolger ihrer Aufgabe gewachsen sein werden, ist einstweilen abzuwarten. Die mit Spannung erwartete Liste der für das Tribunal ausgewählten Richter räumt Zweifel insofern nicht ad hoc aus, denn weder verfügen alle Richter über eine halbwegs solide juristische Ausbildung, noch über die eigentlich dringend nötige Fremdsprachenkompetenz, noch erscheint schließlich in jedem Fall die politische Unabhängigkeit und Unbeeinflussbarkeit zweifelsfrei. Dass einzelne der

Vgl. Laura A. Dickinson, The Promise of Hybrid Courts, AJIL 97 (2003), S. 295 ff.; dezidiert positiv etwa auch Antonio Cassese, The Role of Internationalized Courts and Tribunals in the Fight Against International Criminality, in: Romano (Anm. 32), S. 3 (13): "may prove on of the most effective societal and institutional devices"; eher vorsichtig Alain Pellet, Internationalized Courts: Better than Nothing, ..., in: Romano (Anm. 32), S. 437 ff.

Vgl. Mohamed Othman, East Timor: A Critique of the Model of Accountability for Serious Human Rights and International Humanitarian Law Violations, Nordic Journal of International Law 72 (2003), S. 449 ff.; Sylvia de Bertodano, East Timor, Trials and Tribulations, in: Romano (Anm. 32), S. $79 \mathrm{ff}$.

70

Vgl. etwa die insgesamt wohlwollende Bestandsaufnahme durch Human Rights Watch, Bringing Justice: the Special Court for Sierra Leone. Accomplishments, Shortcomings, and Needed Support, September 2004, Vol. 16, No. 8 (A).

71

Wilhelm Treiber, Der Fall Rote Khmer, DIE ZEIT vom 17.3.2005, S. 15.

72

Häufig hingewiesen wird insbesondere auf die Rolle Chinas, das, zunehmend einflussreich in Kambodscha, erheblichen Druck ausgeübt haben soll, um die Verfahren zu verhindern; vgl. etwa Fawthrop/Jarvis (Anm. 19), S. 234. 
benannten Richter bereits in diversen Gerichtsverfahren problematisch in Erscheinung getreten sind, hat zwangsläufig Kritik provoziert.

Das Tribunal wird nicht nur „hybrid“ mit Blick auf die national-internationale Besetzung der Richterbank sein, es steht auch auf zwei rechtlichen Säulen, einerseits dem nationalen „Law on the Extraordinary Chambers“ (LEC) und andererseits der vertraglichen Vereinbarung zwischen Kambodscha und den Vereinten Nationen (Agreement). Letztere ist ein verbindlicher völkerrechtlicher Vertrag, wie der Verweis auf die Anwendbarkeit der Wiener Vertragsrechtskonvention (Art. 1 Abs. 2 des Agreements) unmissverständlich klar macht. Es stellt sich damit die Frage des Rangverhältnisses zwischen Vertrag und Gesetz und angesichts der politischen Kontroversen zwischen UN und kambodschanischer Regierung verwundert es nicht, dass dieses Thema auch in den Verhandlungen eine Rolle spielte. Das Ergebnis ist so ambivalent wie der gesamte Kompromiss zwischen nationaler Lösung und internationaler Einbindung: einerseits wird wie gesagt die Verbindlichkeit klargestellt, andererseits aber auch in der gleichen Bestimmung betont, dass der Vertrag durch nationales Gesetz umgesetzt wird. Einstweilen erscheint es, als würde das Gesetz den Vertrag recht präzise umsetzen, so dass sich Konflikte vielleicht nicht ergeben. Ausgeschlossen sind Detailprobleme aber nicht. Es stellen sich zudem interessante Verfahrensfragen im Zusammenhang des einigermaßen besonderen Status der ECCC. Ungeklärt ist beispielsweise, wie sich ihr Verhältnis zum Constitutional Council Kambodschas verhält. Zwar hat der Constitutional Council bereits abschließend das Gesetz über die ECCC überprüft, so dass seine Anrufung insoweit nicht mehr in Betracht kommt, sie bleibt aber z.B. dann denkbar, wenn die Verfassungsmäßigkeit kambodschanischen Strafverfahrensrechts in Rede steht und die Frage ist dementsprechend auch schon aufgeworfen worden ${ }^{73}$. Man wird indessen richtigerweise wohl davon auszugehen haben, dass das Regime für die ECCC einen eigenen Mechanismus vorhält, Normdefizite zu bewältigen, nämlich den Rückgriff auf internationale Maßstäbe. Auch bezeichnen Agreement und Gesetz den Instanzenzug von Trial Chamber zu Supreme Court Chamber als abschließend. Verfassungsrechtlich verbleibt gleichwohl eine Unsicherheit, weil die „Richtervorlage“ an den Constitutional Council nun einmal in der Verfassung vorgesehen ist, also im Grundsatz nicht ohne weiteres einfachgesetzlich derogiert werden kann. Es erscheint daher geboten, die ECCC aufgrund ihrer internationalen Zusammensetzung und ihrer sehr speziellen Funktion gar nicht erst als „Courts“ im Sinne von Art. 141 der Verfassung anzusehen. Da hierin eine punktuelle Durchbrechung des Grundsatzes liegt, dass es sich bei den ECCC um einen Bestandteil des nationalen Justizsystems handelt, erscheint allerdings nicht sicher prognostizierbar, dass dieses (wohl sinnvollste) Ergebnis auch in der Praxis erzielt wird. Chambers, unveröffentlichtes Manuskript (Phnom Penh 2005), der freilich - m.E. problematisch - im Ergebnis eine gesetzliche Klarstellung empfiehlt, die den Constitutional Council in den Entscheidungsmechanismus einbezieht. 
Eine wichtige Weichenstellung für das Gericht betrifft naturgemäß die Bestimmung der „Zielpersonen“ des Verfahrens. Die Beschränkung auf die Hauptverantwortlichen des Khmer Rouge Regimes und Taten zwischen dem 17. April 1975 und dem 6. Januar 1979 schließt die Einbeziehung sonstiger Bürgerkriegsparteien (etwa der Lon-Nol-Truppen) und ausländischer Akteure aus. Chinesische, amerikanische, vietnamesische, thailändische und sonstige Verstrickungen in die tragischen Verstrickungen jüngerer Vergangenheit werden insofern zwar mit einiger Sicherheit (von den Rechtsanwälten der Verteidigung) in das Verfahren einfließen, sie bilden aber nicht dessen Gegenstand ${ }^{74}$. Wie viele Khmer Rouge Angehörige letztlich zur Verantwortung gezogen werden, ist Gegenstand von Spekulationen. Sowohl der Begriff der „Senior Leader“ als auch derjenige der „,most responsibles for the serious crimes" ist offen für Interpretation. Die kambodschanische Regierung hat stets betont, um des fragilen Friedens willen nur einen kleinen Kreis von Führungspersonen verfolgen zu wollen, Premierminister Hun Sen hat wiederholt von einer Zahl zwischen vier und zehn gesprochen. Dieser Ansatz bildet auch die Grundlage von Agreement und Gesetz. In einer wissenschaftlichen Untersuchung wurde das Material gegen sieben Personen bereits vorläufig gesichtet und bewertet ${ }^{75}$. Auch wenn es vielleicht bei ambitionierter Ermittlungsarbeit und entsprechender Interpretation der Rechtsgrundlagen einige mehr sein können, bleibt es doch dabei, dass gesetzlich ausschließlich die Verfolgung von Hauptverantwortlichen vorgesehen ist und trotz einiger Unsicherheit im Umgang mit diesem Begriff ist wohl absehbar, dass es sich um eine relativ kleine Zahl an Angeklagten handeln wird ${ }^{76}$. Ein Großteil der Führungselite einschließlich des Hauptverantwortlichen Pol Pot wurde entweder Opfer der parteiinternen Säuberungen oder ist ansonsten verstorben. Für die noch lebenden Zentralfiguren des Regimes lässt sich zum Teil schon angesichts ihres Alters und

In Kambodscha wird dies immer wieder moniert, ist doch der Verweis auf chinesische, vietnamesische oder US-amerikanische Verantwortlichkeiten durchaus populär. Gleichwohl hat die Konzentration auf die inneren Ereignisse durchaus ihre Berechtigung, ist doch der „Vernichtungskrieg“ der Khmer Rouge gegen die eigene Bevölkerung zwar über einige der internationalen Einflüsse mitvorbereitet worden, aber die Verantwortung liegt im Kern doch bei diesem Regime selbst. Und diesbezüglich geht es eben nicht darum, spät den Indochina-Krieg aufzuarbeiten, sondern nur um die besonders brutale Dimension des Regimes der Roten Khmer, die sich mit der Bilanz der Revolutionsregime in Laos und Vietnam eben nicht vergleichen lässt. Die zeitgenössische internationale (Nicht-) Reaktion auf die Verbrechen der Khmer Rouge, so beklagenswert sie ist, mindert deren besondere Schuld nicht.

Heder/Tittemore (Anm. 32). Erfasst sind darin Nuon Chea, Ieng Sary, Khieu Samphan, Ta Mok, Kae Pok (2002 verstorben!), Sou Met und Meah Mut. Premierminister Hun Sen hat diese Publikation als ausländische Einmischung in das kambodschanische Justizsystem kritisiert (Associated Press v. 11.8.2001).

76 An einer flächendeckenden, andere Bürgerkriegsparteien erfassenden sowie die Jahre 1975-1979 extendierenden Aufarbeitung aller geschehenen Verbrechen im Kontext von Khmer Rouge und Bürgerkrieg besteht weder auf Seiten der maßgeblichen Akteure im In- und Ausland ein Interesse (Boyle [Anm. 32], S. 28), es ist auch schwer vorstellbar, wie eine solche Aufgabe technisch und ohne Gefährdung des fragilen politischen Friedens zu leisten sein sollte. 
Gesundheitszustandes die Durchführbarkeit des Verfahrens nicht sicher prognostizieren. Da nach dem Tod von Ta Mok ${ }^{77}$ mit Ausnahme von „Duch“ bislang alle in Rede stehenden Personen auf freiem Fuß sind und sie zudem fast sämtlich nahe der thailändischen Grenze im Bereich von Pailin leben, ist naturgemäß auch nicht auszuschließen, dass sie sich durch Flucht zu entziehen versuchen werden wenn es denn einmal ernst werden würde mit der Strafverfolgung. Als ,kurzgelistet“ für ein Verfahren gelten immerhin:

- „Duch“ (oft auch Deuch; eigentlich: Kaing Khek Iev); geb. 1942; Kommandant des Foltergefängnisses Tuol Sleng; kein Senior Leader der Khmer Rouge, aber relativ offensichtlich ein „Hauptverantwortlicher“ für die Verbrechen des Regimes; seit 1999 in „Untersuchungshaft“ in Phnom Penh.

- Nuon Chea („Brother No. 2“; eigentlich Long Bunruot); geb. 1927; zweiter Mann im System der Khmer Rouge mit zahlreichen Funktionen während der Herrschaftszeit. Kapitulierte 1998; lebt derzeit in Pailin.

- Khieu Samphan, geb. 1929; Staatspräsident im Staat der Khmer Rouge. Kapitulierte 1998; galt lange als das international ,akzeptable“ Gesicht der Khmer Rouge; lebt derzeit in Pailin.

- Ieng Sary (eigentlich Kim Trang); geb. 1927; Vizepremierminister und Außenminister der Khmer Rouge-Regierung. Verfolgungsmöglichkeit zweifelhaft wegen förmlicher Amnestierung im Gegenzug für seine Abkehr von den Khmer Rouge 1996; lebt derzeit in Phnom Penh.

Immerhin einige echte „Senior Leaders“ bzw. „Hauptverantwortliche“, die sich zudem bislang in Kambodscha aufhalten, können also von dem Verfahren vielleicht noch erfasst werden, auch wenn das (abgesehen von „Duch“) durchweg hohe Alter der „Kandidaten“ die Dinge sehr unsicher erscheinen lässt. Dass eine Reihe weiterer Personen in das Blickfeld der Ermittlungen geraten, ist im Übrigen wahrscheinlich, wobei insofern derzeit keine zuverlässigen Prognosen möglich erscheinen, wer im einzelnen erfasst werden könnte ${ }^{78}$.

Im Bereich des materiellen Völkerstrafrechts wird das Gericht eine Frage zu behandeln haben, die für das allgemeine Völkerstrafrecht von Bedeutung ist, nämlich die Voraussetzungen des Völkermord-Tatbestandes. Völkermord (Genozid) gilt als „Crime of Crimes““79

Ta Mok (eigentlich: Chhit Chhoeun); geb. 1925; „,berüchtigter“ Kommandant der Südwest-Zone mit Reputation für Massensäuberungen; zahlreiche Partei- und Militärämter; später exzellente Kontakte zum thailändischen Militär; letzter Führer der Khmer Rouge nach Verhaftung Pol Pot's 1997; seit 1999 bis zu seinem Tod im Juli 2006 in „Untersuchungshaft“ in Phnom Penh; galt als Kandidat für eine der ersten Anklagen vor den ECCC.

Für eine Liste von "Senior Leaders" sowie "Hauptverantwortlichen" für die Verbrechen auch Fawthrop/Jarvis (Anm. 19), S. 254 ff. wobei die Auflistung eindrucksvoll belegt, dass der Großteil der Verantwortlichen inzwischen gestorben ist. 
oder „ultimate crime“ ${ }^{80}$ und manchen erscheint es wichtig, genau diese Qualifikation zu erreichen ${ }^{81}$. Mit Blick auf die Herrschaft der Khmer Rouge wurden die Verbrechen der Khmer Rouge relativ früh von allen Seiten mit dem Begriff „Genozid“ verbunden. Die kambodschanische Regierung selbst qualifizierte die Taten der Khmer Rouge ab 1979 durchgehend als Genozid und die Verurteilung von Pol Pot und Ieng Sary in dem Verfahren 1979 erfolgte einzig wegen Genozids (freilich auf der Grundlage einer flexibel und ad hoc an die Verbrechen der Khmer Rouge angepassten Definition ${ }^{82}$ ). Auch in den Vereinten Nationen wurde bisweilen der Begriff benutzt ${ }^{83}$. Ein führendes deutsches Völkerrechtslehrbuch geht unbesehen von Genozid aus ${ }^{84}$. In den USA wurde gar ein „Cambodian Genocide Justice Act ${ }^{\star 85}$ erlassen und an der Yale Law School etablierte man ein langjähriges „Cambodian Genocide Program“, das erheblich zur Tatsachenermittlung beigetragen hat, aber gewissermaßen im Namen einen Nachweis für das Vorliegen von Völkermord versprochen hat. Völkerstrafrechtlich liegt das Problem nun darin, dass der Genozid eine definierte Straftat ist, die nicht einfach den massenhaften Mord und auch nicht den massenhaft politischen Mord erfasst, sondern an den Willen zur Auslöschung eines „Volkes“ bzw. einer in bestimmter Weise identifizierbaren Gruppe anknüpft ${ }^{86}$. Die Völkermord-Konvention der Vereinten Nationen vom 9.12.1948 ${ }^{87}$ ist unmittelbare Reaktion auf den deutschen „Holo-

Payam Akhavan, Enforcement of the Genocide Convention: A Challenge to Civilization, Harvard Human Rights Journal 8 (1995), S. 229.

Vgl. eingehend schon Hurst Hannum, International Law and Cambodian Genocide: The Sounds of Silence, Human Rights Quarterly 11 (1989), S. 82 ff.; Gregory H. Stanton, The Cambodian Genocide and International Law, in: Ben Kiernan (ed.), Genocide and Democracy in Cambodia (1993).

Nach Art. II des "Decree Law No. 1" vom 15. 7.1979 waren erfasst: "planned massacres of groups of innocent people; expulsion of inhabitants of cities and villages in order to concentrate them and force them to do hard labour in conditions leading to their physical and mental destruction; wiping out religion; destroying political, cultural and social structures and family and social relations." (Zitiert bei Fawthrop/Jarvis (Anm. 19), S. 223).

Revised and Updated Report on the Question of the Prevention and Punishment of the Crime of Genocide Prepared by Mr. B. Whitaker (U.N. Doc. E/CN/Sub.2/1985/6 [1985], 9 f.).

Alfred Verdross / Bruno Simma, Universelles Völkerrecht (3. Auflage 1984), § 110, nach denen „feststeht, dass sich die Regierung Pol Pot des Genozids an der eigenen Bevölkerung schuldig gemacht hat."

Bereits 1987 hatte der US-Gesetzgeber ,autoritativ“ festgestellt: „The persecution of the Cambodian people under the Khmer Rouge rule, (when) the bulk of the Khmer people were subjected to life in an Asian Auschwitz, constituted one of the clearest examples of genocide in recent history." (United States Foreign Relations Authorization Act, Fiscal Years 1988 and 1989, Pub.L No. 100204, § 906).

Zum Genozid-Tatbestand s. neben Schabas (Anm. 78) z.B. Werle (Anm. 53), S. 198 ff.; Antonio Cassese, International Criminal Law, 2003, S. 96 ff.; Bantekas/Nash (Anm. 58), S. 358 ff. Vgl. auch die instruktive und mit speziellem Fokus auf Kambodscha geschriebene Darstellung von Ratner/Abrams, (Anm. 63).

UNTS 78, 277, in Kraft seit dem 12.1.1951; zum Ratifikationsstand www.preventgenocide.org. 
caust" an den europäischen Juden und der seither wohl klarste Fall von Genozid-Verbrechen findet sich in Ruanda, wo die Minderheiten-Ethnie der Tutsi ein halbes Jahrhundert später einer Vernichtungskampagne ausgesetzt war $^{88}$, wobei sich die internationale Gemeinschaft, unwillig einzugreifen, diese Qualifikation zunächst zu vermeiden trachtete ${ }^{89}$. Zwar sind nicht alle Details um den Genozid-Tatbestand geklärt, unumstritten ist aber, dass ein Zentralangriff auf die eigene Bevölkerung, wohl nicht um sie auszulöschen, sondern um sie zu disziplinieren, den etablierten Kriterien nicht entspricht ${ }^{90}$. In diesem Sinne lässt sich der Genozid im Sinne eines juristischen Tatbestandes auch nicht einfach durch politischen bzw. journalistischen Sprachgebrauch (,If it is awful, it must be genocide “91 ) herbeireden. Der Versuch, den juristischen Genozid-Begriff über den Rekurs auf moderne völkergewohnheitsrechtliche Entwicklung zu erweitern ${ }^{92}$, führt mit Blick auf das Khmer Rouge Tribunal wohl ebenfalls nicht weiter, weil dessen rechtliche Grundlagen für die Definition ausdrücklich auf die Völkermordkonvention verweisen. Legt man deren Kriterien zugrunde, mag es zwar sein, dass sich ein Genozid-Vorwurf mit Blick auf Minderheiten und Gruppen wie Vietnamesen, Chinesen, muslimische Cham oder das buddhistische Mönchtum verdichten lässt. Hierzu ist viel argumentiert worden und vielleicht lassen sich, trotz mancher Schwierigkeiten, Genozid-Elemente im System der Khmer Rouge im Gerichtsverfahren insofern auch nachweisen ${ }^{93}$. Prozessual wird das Problem aber dann ggf. immer noch darin liegen, sie den konkret vor Gericht stehenden Personen individuell zuzurechnen, denn auch im Internationalen Strafrecht geht es nicht um die Existenz eines irgendwo entworfenen Gesamtplans allein, sondern um individuelle Verantwortung. Es bleibt im Übrigen jedenfalls der Befund, dass sich über solche Ansätze in der Gesamtbe-

Vgl. insbesondere Prosecutor v Akayesu, Case No. ICTR 96-4-A, Urteil vom 2.9.1998, Appeal Chamber: 1.6.2001 sowie das Verfahren gegen den früheren Premierminister Ruandas Prosecutor v Kambanda, Case No. ICTR 97-23-A, Urteil vom 4.9.1998; Appeal Chamber: 19.10.2000. Die (Jugoslawien betreffenden) deutschen Grundsatzentscheidungen sind BGHSt 45, 64 / BVerfG NJW 2001, 1848 - Nicola Jorgic.

Am 25. Mai 1998 bekannte US-Präsident Bill Clinton in einer Rede auf dem Flughafen von Kigali: "We did not immediately call these crimes by their rightful name: genocide." (New York Times v. 26.3.1998, A 10).

Ein kambodschanischer Gesetzentwurf aus dem Jahr 1999 versuchte dementsprechend, die Kriterien des Genozidtatbestandes um folgende Gruppenmerkmale zu erweitern: "wealth, level of eduction, sociological environment (urban/rural), allegiance to a political system or regime (old people/new people), social class or social category (merchant, civil servant etc.)“; zitiziert nach William A. Schabas, Problems of International Codification - Were the Atrocities in Cambodia and Kosovo Genocide?, New England Law Review 35 (2001), S. 287 (293 in Fn. 23). Helen Fein, zitiert bei Patricia Wald, Judging Genocide, in: Justice Initiatives (Anm. 32), S. 85.

Vgl. Beth Van Schaack, The Crime of Political Genocide: Reparing the Genocide Convention's Blind Spot, Yale Law Journal 106 (1997), S. 2259 ff.

Schabas (Anm. 89) weist freilich zu Recht darauf hin, dass beispielsweise die Zerstörung von Kirchen und Moscheen oder das Verbot der Benutzung eigener Sprache noch nicht den GenozidTatbestand erfüllt, weil ein ,kultureller Genozid“ im Allgemeinen bewusst nicht erfasst wurde. 
trachtung die eigentliche verbrecherische Qualität des Regimes der Khmer Rouge nicht vollständig oder auch nur überwiegend erfassen lässt. Die meisten Opfer gehörten eben keiner der genannten Minderheiten an und die alleinige oder auch nur schwerpunktmäßige Konzentration auf diese Minderheiten würde dem Andenken an die Mehrheitsopfer nicht gerecht. Ein Regime, das in weniger als vier Jahren zwischen 20 und 30 Prozent seiner eigenen Bevölkerung im wahrsten Sinne „wahllos“ tötet bzw. im Rahmen seiner gesellschaftspolitischen Maßnahmen sterben lässt und dabei sukzessive an Brutalität zunimmt, macht sich wohl journalistisch gesprochen des versuchten „Auto-Genozids“ schuldig ${ }^{94}$. Der Vorgang lässt sich aber eben nicht ohne weiteres juristisch sub specie „Genozid“ im Völkerstrafrecht fixieren ${ }^{95}$. Für das Völkerrecht und für Kambodscha wäre es kein guter Dienst, wenn sich die Ermittlungen (ggf. in Reaktion auf Erwartungshaltungen der interessierten Öffentlichkeit) in der Konsequenz letztlich nur auf die Minderheiten stürzen würden, denn Opfer waren vor allem auch die „,normalen“ Kambodschaner selbst. Völkerstrafrechtlich sind es wohl Verbrechen gegen die Menschlichkeit, die insoweit überwiegend in Rede stehen. Das macht sie nicht entschuldbarer und auch nicht weniger strafbar ${ }^{96}$.

Während das vom Gericht anzuwendende materielle Strafrecht im Übrigen relativ klar zu sein scheint, sind erhebliche Probleme im Feld des Verfahrensrechts abzusehen. Insbesondere für einen der zentralen Kandidaten, den Khmer Rouge-Außenminister Ieng Sary, stellt sich im Themenfeld der Verfahrensvoraussetzungen das Problem der Geltung und Reichweite einer ihm 1996 erteilten königlichen Amnestie, die er im Gegenzug für den Rückzug aus dem Bürgerkrieg erhalten hatte. Konkret erfasste diese Amnestie seine Verurteilung zum Tode im Jahr 1979 sowie (für den Kontext des Khmer Rouge Tribunals nicht relevant) Verstöße gegen das Khmer Rouge-Parteiverbot aus dem Jahr 1994. Mit Blick auf seine Verantwortlichkeit in den Jahren 1975 bis 1979 wird zum Teil geltend gemacht, die Amnestie erfasse nur das Genozid-Delikt, auf dem das Todesurteil beruhte, so dass die Verfolgung weiterer Straftatbestände nach wie vor in Betracht komme. In den Verhandlungen um ein Tribunal machten die Vereinten Nationen grundsätzlicher geltend, auch vorangegangene Amnestien könnten internationale Verbrechen nicht erfassen. Die kambodschanische Regierung, für die sich die Amnestie zugunsten von Ieng Sary als wesentlicher Schritt zur Beendigung des Bürgerkriegs darstellt (ein Befund, der auch von unabhängigen Historikern geteilt wird), weigerte sich indessen, dessen in aller Förmlichkeit vollzogenen

Der treffende Begriff wurde von dem französischen Indochina-Experten Jean Lacouture im März 1977 in einer Rezension des Buches von Paunchaud (Anm. 15) im New York Review of Books benutzt: „Ordinary genocide (if one can ever call it ordinary) usually has been carried out against a foreign population or an internal minority.... The new masters of Phnom Penh have invented something original: auto-genocide. After Auschwitz and the Gulag, we might have thought this century has produced the ultimate in horror, but we are seeing the suicide of a people in the name of revolution ..." (zitiert bei Shawcross, The Quality of Mercy [Anm. 13], S. 63). 
Amnestierung zu invalidieren. Die Vereinbarung zwischen der kambodschanischen Regierung und den Vereinten Nationen und das nationale Gesetz weisen die Rechtsfrage in einem Formelkompromiss der Beurteilung ausdrücklich den ECCC zu. Außerdem verpflichtet sich die Regierung Kambodschas in der Vereinbarung, keine weiteren Amnestien zu beantragen (für deren Gewährung verfassungsrechtlich der König kompetent ist). Mit Blick auf Ieng Sary könnte sich die Amnestiefrage aber politisch und rechtlich als schwierig erweisen, was sich schon daran zeigt, dass sich literarische Analysen bislang in aller Regelmäßigkeit um eine Bewertung herumdrücken ${ }^{97}$.

Hinsichtlich der eigentlichen Verfahrensfragen verweist das Gesetz im Einklang mit der Vereinbarung grundsätzlich auf das kambodschanische Recht, sieht aber gleichzeitig vor, dass im Fall von Defizitzuständen im nationalen Recht internationale Standards herangezogen werden mögen. Diese scheinbar schlichte Regelung wird Grundlage erheblicher Probleme sein, denn weder genügt kambodschanisches Strafverfahrensrecht internationalen Standards, noch sind die letzteren selbst wirklich präzise definiert: Bezüglich des kambodschanischen Strafverfahrensrechts gibt es bislang weder eine geltende Strafprozessordnung, wie man es in einem dem Civil-Law-Rechtskreis zugehörenden Staat typischerweise erwartet $^{98}$, noch auch nur ein geschlossenes System von speziellen Gesetzen ${ }^{99}$; es gibt keine standardisierte Rechtsprechungspraxis und keine substanzielle literarische ErschlieBung ${ }^{100}$. Es gibt zusammengefasst kaum etwas, das Strafverfahren in ihren Regeln verlässlich machen könnte und es ist allgemein anerkannt, dass die kambodschanische Strafverfahrenspraxis bislang in keinerlei Hinsicht internationalen Standards genügt und insbesondere

Signifikant etwa bei Heder/Tittemore (Anm. 32), die in ihrer Analyse der Verantwortlichkeit von Ieng Sary das Amnestieproblem nicht einmal erwähnen; vgl. auch Shraga, Meijer und Swart in: Romano (Anm. 32), S. 30 f ., 214 f., 313 f., die zwar sämtlich das Amnestieproblem bezüglich Ieng Sary aufwerfen, sich aber ebenfalls sämtlich einer Stellungnahme enthalten; kritisch - aber nicht sehr klar in der Gedankenführung - Boyle (Anm. 32), S. 29 ff.

Ein im Rahmen französischer Entwicklungskooperation vorbereiteter Gesetzentwurf für eine Strafprozessordnung wurde dem Vernehmen nach Ende August 2006 vom Kabinett gebilligt und sollte nun dementsprechend alsbald ins parlamentarische Verfahren gehen. Wie schnell der Entwurf zum Gesetz wird, bleibt abzuwarten.

Hinsichtlich des Strafverfahrens stehen in der Praxis zwei rudimentäre bzw. unzeitgemäße Gesetze in offener Regelungskonkurrenz, vgl. einerseits die von der UN-Übergangsverwaltung erlassenen „Provisions Relating to the Judiciary and Criminal Law and Criminal Law and Procedure Applicable in Cambodia during the Transitional Period“v. 10.9.1992 (sog. „UNTAC-Law“) sowie andererseits das von der Nationalversammlung des State of Cambodia erlassene „Law on Criminal Procedure“ vom 28.1.1993. Auf welches Gesetz zurückgegriffen wird, entscheidet sich in der Praxis nicht nach juristischen Regeln, sondern richterlicher Beliebigkeit, vgl. nur Ratner/ Abrams (Anm. 63), S. 312.

100

Vgl. aber immerhin Stuart Coghill, Resource Guide to the Criminal Law of Cambodia (2000). 
von politischer Unabhängigkeit nicht zu reden ist ${ }^{101}$. Hinsichtlich der internationalen Standards ist allerdings ebenfalls nicht von einer sicheren Situation zu sprechen. Es mag sich gegebenenfalls aufdrängen, die Regeln des ICC-Statuts heranzuziehen, zumal Kambodscha dem Internationalen Strafgerichtshof früh beigetreten ist. Ob sich aber die hoch gehängten Standards, wie sie das ICC-Statut prägen, in der Praxis eines - wenn auch internationalisierten - kambodschanischen Gerichts durchhalten lassen, erscheint zweifelhaft. Ein Quervergleich zum Verfahrensrecht in Sierra Leone mag sich jedenfalls anbieten. Einstweilen wird wohl zu Recht geltend gemacht, dass die Regelung gewissermaßen in einen Zustand interpretatorischer Beliebigkeit führt ${ }^{102}$. Auch wenn man grundsätzlich davon ausgeht, dass es immer zu den richterlichen Aufgaben gehört, mit nicht ganz präzisen Vorgaben umzugehen, erscheint der vorliegende Zustand doch und zumal im Angesicht der Zusammensetzung des Richterkollegiums besonders problematisch. Ein auf möglicherweise sehr unterschiedlichen Vorstellungen agierendes Richterkollegium wird sich unzureichendes nationales Recht ansehen und ergänzend wenig präzise allgemeine internationale Standards heranziehen. All dies wird geschehen in Strafverfahren, in denen, weil sie ja Hauptverantwortliche für die Verbrechen der Khmer Rouge sind, voraussichtlich für jeden Angeklagten die Höchststrafe „lebenslänglich“ in Rede stehen wird. Festzuhalten ist im Übrigen, dass es mitnichten nur um Verfahrensrechte der Angeklagten geht, sondern auch z.B. um Zeugenschutz und ähnliches. Soweit Prognosen überhaupt möglich sind, scheint es absehbar, dass das Verfahrensrecht „das“ juristische Hauptproblem des Verfahrens wird. Konkret wurde im Vorfeld etwa über die Frage diskutiert, ob die Beschuldigten das Recht auf einen internationalen Rechtsbeistand haben müssen. Trotz einer beiläufigen Erwähnung internationaler Rechtsbeistände im Agreement zwischen Regierung und UN bestand Sorge, dass gemäß „,normalem“ kambodschanischem Recht nur Kambodschaner als Verteidiger tätig werden können ${ }^{103}$. Angesichts der Tatsache, dass sich die Angeklagten einer geballten Macht internationaler Strafverfolgungs- und Richterkompetenz ausgesetzt sehen werden, erschien das freilich - vorsichtig formuliert - ungereimt. Inzwischen hat das Büro der ECCC offiziell die Einrichtung eines eigenen Verteidigerbüros angekündigt, das mit ausländischen Verteidigern besetzt sein wird ${ }^{104}$. Das vielleicht nur scheinbare Rechtsproblem ${ }^{105}$ ist damit von

101

Gerade in jüngerer Zeit waren relativ klare Instrumentalisierungen der Justiz zu verzeichnen mit rechtlich mehr als dubiosen Verfahren gegen eine Reihe von Oppositionspolitikern und Menschenrechtsaktivisten, die teilweise in Inhaftierungen und Verurteilungen mündeten, weltweiten Protest auslösten und sodann Anfang 2006 (die politische Steuerbarkeit erneut belegend) fast schlagartig ihr Ende fanden; vgl. im Überblick ADHOC Human Rights Report 2005, Phnom Penh 2006, S. $55 \mathrm{ff}$.

Sarah Williams, The Cambodian Extraordinary Chambers - A Dangerous Precedent for International Justice?, ICLQ 2004, S. 227 (242).

103 Law on the Cambodian Bar, Art. 3; vgl. auch Caitlin Reiger, Marrying International and Local Justice: Practical Challenges Facing the Khmer Rouge Tribunal, in: Justice Initiatives (Anm. 32), 104 S. 97 (105).

Cambodia Daily vom 8. July 2006, S. 3. 
offizieller Seite jedenfalls adressiert, wobei ggf. noch die Frage des Rechts auf einen ausländischen Verteidiger „eigener Wahl“ verbleibt.

Angesichts der erheblichen zeitlichen Distanz von wohl um die dreißig Jahren zwischen den in Rede stehenden Taten und einem Verfahren erscheint es nahe liegend, große Schwierigkeiten bei der Beweisführung zu erwarten, weil Dokumente häufig verschwunden sind, mehr aber noch, weil Zeugen häufig nicht mehr greifbar sind und ggf. nur eine unpräzise Erinnerung haben werden ${ }^{106}$. Allerdings hat das „,rechtlose“ System der Khmer Rouge trotz systematischer Vernichtung zum Ende der Regimezeit doch eine erstaunlich große Zahl an Dokumenten hinterlassen, die etwa die verbrecherischen Tätigkeiten im Verhörund Foltergefängnis Tuol Sleng sowie des Staatssicherheitsdienstes Santebal (,Bewahrer des Friedens“) dokumentieren ${ }^{107}$ und ein überwiegend ausländisch finanziertes ,Documentation Center of Cambodia“ (,DC-Cam“) hat seit über zehn Jahren systematisch Daten zur Dokumentation der Verbrechen während der Zeit der Roten Khmer gesammelt ${ }^{108}$. Seit Jahren publiziert das Documentation Center seine Forschungsergebnisse in Monographien und einer eigenen Zeitschrift (,Searching for the Truth“), die im ganzen Land zirkuliert. Entstanden ist eine Sammlung von Beweismaterial, die so aufbereitet wohl noch keinem internationalen oder semi-internationalen Strafgericht bereits $\mathrm{zu}$ Arbeitsbeginn zur Verfügung gestanden hat. Nicht nur wurden die verfügbaren Dokumente systematisch gesammelt, es wurde z.B. auch gezielt eine Bestandsaufnahme der über ganz Kambodscha verstreuten Massengräber unternommen. Die verbrecherische Qualität des Regimes dürfte auf der Grundlage dieser Befunde zweifellos sein, so dass es in den Verfahren vor allem darum gehen wird, die einschlägigen Delikte den konkreten Angeklagten zuzurechnen. Im Vergleich zu manchen sonstigen internationalen und gemischten Gerichten dürfte die Beweissituation insofern dadurch vereinfacht sein, als nach Gesetzeslage nur Hauptverantwortliche in Rede stehen, also nur „Verdächtige“, für die typischerweise die Dokumentenlage weit besser ist als für lediglich ausführende Täter. Einigermaßen offensichtlich ist

Die gebotene „agreement-konforme“ Auslegung des Gesetzes über die Extraordinary Chambers sollte m.E. relativ problemlos die Möglichkeit der Zulassung ausländischer Rechtsanwälte in diesen Verfahren (im Sinne einer lex specialis zum Law on the Cambodia Bar) ergeben.

Vgl. zu Beweisfragen auch Julia Fromholz, Proving Khmer Rouge Abuses: Uses and Limitations of the Available Evidence, in: Ciociari (Anm. 32), S. 107 ff. sowie schon Ratner/Abrams (Anm. 63), S. $325 \mathrm{ff}$.

107

Vgl. George Chigas, The Trial of the Khmer Rouge: the role of the Tuol Sleng and Santebal archives, Harvard Asia Quarterly 2000 vol. IV (http://www.fas.harvard.edu/\%7Easiactr/haq/ index.html).

108 Das Documentation Center of Cambodia war zunächst „Field Office“ des Yale Genocide Project, das die Universität Yale in Ausführung des US-amerikanischen Cambodian Genocide Act aufgelegt hatte. Seit 1997 ist DC-Cam eine rechtlich unabhängige kambodschanische Nichtregierungsorganisation. Inzwischen ist es auch im Bildungsbereich tätig. Für Informationen siehe auch die Internetseite http://www.dccam.org./ 
jedenfalls, dass z.B. die Verantwortlichkeit von „Duch“, dem Leiter des Foltergefängnisses S 21, keine größeren Probleme aufwerfen wird. „Duch“ hat sich zu seinen Taten im Übrigen (als einziger der prognostizierten Angeklagten) im Grundsatz bekannt ${ }^{109}$.

\section{Eine Chance für Recht und Gerechtigkeit}

Der Blick auf das anstehende Tribunal sollte nicht von Beginn an nur durch die Analyse denkbarer Schwierigkeiten und Probleme geprägt sein, sondern auch die positiven Perspektiven ernst nehmen. Zu Recht wurde insofern in jüngerer Zeit häufig betont, dass ein nicht perfektes Verfahren wohl besser ist als keines. Die Khmer Rouge gehören zu denjenigen Regimen des 20. Jahrhunderts, in denen über Jahre hinweg ein Totalexzess staatlicher Macht stattgefunden hat, der auch international nicht gestoppt wurde. Die Art und Weise, wie die Verantwortlichen nach ihrer Vertreibung noch hofiert wurde, ist ein nur über die Eigengesetzlichkeiten des Kalten Krieges erklärlicher, aber auch insofern nicht entschuldbarer Politikskandal. In diesem Sinne ist ein solches Verfahren indirekt auch für die internationale Gemeinschaft eine Konfrontation mit der Vergangenheit eigener „Realpolitik“, die Kambodscha vor, während und nach der unmittelbaren Herrschaftszeit der Khmer Rouge für lange Zeit zu einem Spielball internationaler Politik gemacht hatte. Dass die internationalen Verstrickungen im Übrigen auch praktische Verfahrensfragen etwa mit Blick auf Auslieferung von Tatverdächtigen oder die Zurverfügungstellung von Dokumenten implizieren können, liegt auf der Hand ${ }^{110}$.

Für Kambodscha bringt das Verfahren zunächst die Chance einer wenn auch späten und unvollständigen Gerechtigkeit. Die kambodschanische Bevölkerung will nach allen Erhebungen mit Mehrheit eine solche Aufarbeitung ${ }^{111}$, auch wenn insbesondere im kambod-

109

Zu Duch eingehend Nic Dunlop, The Lost Executioner (2005). Dunlop spürte Duch, der unter falschem Namen im Westen Kambodschas lebte, Anfang 1999 in einem Dorf in der Nähe der Khmer Rouge Hochburg Pailin nahe der Grenze zu Thailand auf, wo Duch, zum Christentum konvertiert, als Lehrer arbeitete, nachdem er zuvor in einem thailändischen Camp auch für eine amerikanische Flüchtlingsorganisation tätig gewesen war sowie eng mit „World Vision“ zusammen gearbeitet hatte. Nach dem Interview mit Dunlop verschwand Duch für kurze Zeit und stellte sich dann den kambodschanischen Behörden.

Dazu Ratner/Abrams (Anm. 63), 326 f., die verbreitet vorfindliche Erwartungen wiedergeben, wenn sie im Angesicht historischer Rahmenbindungen Vietnam für potentiell kooperationswillig, Thailand eher für unsicher und China sowie Laos für eher kooperationsunwillig halten.

111 Vgl. zuletzt etwa die Ergebnisse des vom Khmer Institute of Democracy durchgeführten „Survey on the Khmer Rouge Regime and Khmer Rouge Tribunal“, Phnom Penh 2004, S. 6 (mit Hinweisen auf sonstige Umfragen). Umfänglicher zu Erwartungshaltungen in der Bevölkerung auch Laura McGrew, Truth, Justice and Peace in Cambodia. 20 Years after the Khmer Rouge, Phnom Penh 2000. Angesichts der vorhandenen Erhebungen jedenfalls problematisch Oskar Weggel, Das Khmer-Rouge-Tribunal - Auftakt zur Vergangenheitsbewältigung? Südostasien aktuell 4/2004, S. 
schanischen Westen, wo bis vor einigen Jahren der Bürgerkrieg herrschte und noch heute zahlreiche ehemalige Khmer Rouge leben, naturgemäß auch Bedenken bestehen. Die Staatsführung hat im Rahmen der Verhandlungen mit den Vereinten Nationen sowie der flankierenden nationalen Gesetzgebung die juristische Aufarbeitung zur offiziellen Politik gemacht und dies sollte, diverser Verzögerungen zum Trotz, bis zum Antritt des Gegenbeweises ernst genommen werden. Es geht im Übrigen richtig betrachtet nicht so sehr darum, ob die Regierung das Verfahren aus tiefer Überzeugung ,will“, sondern eher darum, ob sie es als Notwendigkeit akzeptiert, in der vereinbarten Form unterstützt und seine Unabhängig respektiert. Man sollte insoweit die Standards auch nicht höher hängen als bei anderen internationalen oder internationalisierten Verfahren, wo die Regierungen vor Ort bekanntlich auch nicht immer die glühendsten Verfechter der Idee waren bzw. sind. Auch wäre es verfehlt, nach den Erfolgen des Verfahrens nur in seinen abschließenden Entscheidungen zu suchen. Rechtsprechung erfüllt generell Funktionen über die Klärung konkreter Streitfälle hinaus und für Gerichte der hier in Rede stehenden Art gilt dies natürlich in besonderem Maße $\mathrm{e}^{112}$. Wilhelm Treiber hat zu Recht auf die Bedeutung hingewiesen, die es für den außergerichtlichen Diskurs hat ${ }^{113}$. Justizförmliche Mechanismen sind wichtig und werden zunehmend erwartet ${ }^{114}$, sie sind jedoch nur eine Facette der notwendigen Auseinandersetzung mit der Vergangenheit. Immerhin können sie, und dies scheint in Kambodscha recht deutlich, einen allgemeinen Diskurs stimulieren. Schon im Vorfeld hat die schlichte Aussicht auf das Khmer Rouge Tribunal zahlreiche Nichtregierungsorganisationen darin motiviert, die Befindlichkeiten in der kambodschanischen Bevölkerung mit Blick auf das düsterste Kapitel der Vergangenheit ihres Landes zu erkunden und Kenntnisse über diese Vergangenheit zu vermitteln. Zuletzt hat die größte Tageszeitung des Landes angekündigt, in Zusammenarbeit mit dem Cambodian Documentation Center einen regelmäßigen „Khmer Rouge Watch“ einzurichten ${ }^{115}$. Gelegentliche Forderungen nach einer Truth and Reconciliation Commission sind zwar in Kambodscha nicht auf größere Resonanz gesto-

10 (14), nach dem die überwiegende Mehrheit der Bevölkerung aus ,theradava-buddhistischen und aus animistischen Erwägungen nicht noch einmal den Staub der Vergangenheit aufwirbeln und die Geister der Ermordeten erneut wecken" wolle.

Eine treffende Zusammenfassung von Zielprojektionen findet sich bei Sok Sam Oouen, Direktor der NGO "Cambodian Defenders Project” (Phnom Penh Post v. 4.2.2000, S. 13): „If this tribunal is conducted well, in accordance with international standards and principles of fair trial, it can have at least four following good results: first, to provide justice who are victims of this regime; second, to heal Cambodian society and end nightmares of Cambodian victims; third, to find the truth, so that Cambodians and the rest of the world can know why 1.7 million people died; and finally, I hope that this tribunal can serve as a model to show Cambodian people what the principles of a fair trial are."

113

Der Fall Rote Khmer, Die ZEIT, 17.3.2005, S. 15; vgl. auch Tracey Gurd, Outreach: A Key to Success, in: Justice Initiatives (Anm. 32), S. 139 ff.

114 Vgl. zum Thema statt vieler Ruti G. Treitel, Transitional Justice (2000) sowie instruktiv auch 
ßen, schon die Diskussion um die Gerichtsverfahren hat aber zu solcher Wahrheitssuche auf der Basis vielfältiger Einzelprojekte beigetragen. Institutionen wie das Documentation Center of Cambodia schöpfen ihre Kraft zwar unübersehbar gerade auch aus der Perspektive, dass die gesammelten Daten auch in einem Gerichtsverfahren nutzbar gemacht werden können, sie tragen aber mit ihren Erhebungen schon unabhängig von solcher justizieller Aufarbeitung zu historischer Wahrhaftigkeit bei. Betrachtet man die aktuelle Diskussion, wird man im Sinne einer gesellschaftlichen Aufarbeitung der Zeit der Khmer Rouge feststellen können, dass das Tribunal schon vor Arbeitsbeginn begonnen hat, einen Teil seiner Funktion zu erfüllen.

Auch wenn es bei dem Verfahren natürlich zunächst und vor allem um Gerechtigkeit für die Opfer des Khmer Rouge Regimes geht, sollten mögliche Begleiteffekte nicht untergewichtet werden. Für Rechtssystem und Rechtskultur Kambodscha stellt das Verfahren eine Chance dar, sich fortzuentwickeln ${ }^{116}$. Diese Fortentwicklung ist, auch aus der Perspektive des internationalen Menschenrechtsschutzes, anerkanntermaßen dringend notwendig, denn Kambodscha gehört rechtlich zweifellos zu den „least developed countries“ weltweit. Ein Großteil der Richterschaft ist ohne juristische Ausbildung, Rechtsgrundlagen sind notleidend, Gerichtsentscheidungen ergehen üblicherweise ohne Begründung und bleiben fast vollständig unpubliziert. Korruption und politische Beeinflussung, letztere nicht selten wohl auch im Wege „,vorauseilenden Gehorsams“ einer an politische Oberherrschaft gewöhnter Richter, scheinen endemisch ${ }^{117}$. Eine Kultur der Straflosigkeit (impunity“) ${ }^{118}$ kennzeichnet ein Rechtssystem, das bislang weder internationalen Standards noch den Maßgaben der eigenen nationalen Verfassung entspricht. Die ECCC werden einige der Defizite erneut in das nationale und internationale Bewusstsein rufen und sie mögen im günstigen Fall vielleicht Momentum für eine substanzielle Verbesserung sein. Sie können beiläufig die Reformkräfte unterstützen, die an einer schrittweisen Verbesserung des Systems arbeiten. tribunal needs more than money, Bangkok Post v. 12.7.2005, S. 12; Williams (Anm. 102), S. 245.

Statt aller Ratner/Abrams (Anm. 63): „The Cambodian judiciary presently meets none of the key criteria for a fair and effective judiciary - a workable legal framework; a trained cadre of legal advocates, decision-makers, and investigators; adequate infrastructure; and a culture of respect for due process. Instead, like those of many countries emerging from national traumas, it is a disorganized, ineffective, and unfair system, currently failing to mete out criminal justice, notwithstanding the laudable aid programs of NGOs, the UN, and foreign governments."

118 Aus menschenrechtlicher Perspektive äußern sich hierzu alle aktuellen Berichte, vgl. etwa ADHOC, Human Rights Situation Report 2004 (Januar 2005); LICHADO, Cambodia Human Rights Report 2004 (Juli 2004); LICHADO, Human Rights in Cambodia: The Façade of Stability (May 2006); Report of the Special Representative of the Secretary General for Human Rights in Cambodia, Peter Leuprecht, E/CN.4/2005/116 vom 20.12.2004. 


\section{Der Vorhang auf und alle Fragen offen}

Noch ist der Vorhang für die Verfahren nicht offen und er wird auch nicht wie ursprünglich vorgesehen in dem traditionsreichen Chaktomuk-Theater in der Mitte Phnom Penh geöffnet werden, wo 1979 bereits das zumeist als Schauprozess qualifizierte Verfahren gegen Pol Pot und Ieng Sary stattgefunden hatte, sondern in modernen, zur Zeit noch im Rohbau befindlichen Einrichtungen am Stadtrand. Auch in der Sache ist zu hoffen, dass es kein „Theaterstück“ wird. Es geht um Grundfragen der Staats- und Rechtskultur und den Kambodschanern ist zu wünschen, dass das Verfahren, für das sich noch viele Risiken stellen werden, zumindest einen Teil der Erwartungen erfüllt und nicht in Enttäuschung mündet. Nach 1979 hat es siebzehn Jahre gebraucht, um den Gedanken einer rechtsstaatlichen juristischen Aufarbeitung der Verbrechen der Khmer Rouge aufzugreifen und seitdem ist erneut eine Dekade vergangen, ihn der Realisierung immerhin nahe zu bringen. Es wäre einzigartig, solche Verbrechen so spät in einem speziell eingerichteten Gericht zu verhandeln. In Kambodscha hat man häufig gerne alle Verantwortung bei dem „Haupttäter“ Pol Pot abgeladen. Die Tatsache, dass „Brother Nr. 1“ nach seinem Tod nicht mehr für ein Verfahren zur Verfügung steht, lenkt nun zwangsläufig den Blick auf die Mitverantwortung sonstiger Systemträger, ohne die kein Diktator weltweit je agieren konnte. Das „Eichmann'sche“ Verteidigungsargument, man habe nur Befehle befolgt, zieht nicht mehr; das Böse wird bestraft, auch wenn es banal ist ${ }^{119}$. Ob die von den ECCC angeklagten Personen begangenen Taten dabei letztlich als „Genozid“ oder „Verbrechen gegen die Menschlichkeit“ zu bezeichnen sein werden, erscheint sekundär, wichtig ist, dass die Verantwortung überhaupt klargestellt wird. Mit Blick auf eine Zukunft praktischen internationalen Strafrechts geht es im Übrigen darum, in einem durchaus schwierigen Umfeld die Entwicklung eines Verfahrensmodells zu bewerten, das stärker den lokalen Gegebenheiten ausgesetzt ist als die „Mega“-Gerichte nach dem Muster der Jugoslawien- und Ruanda-Tribunale, die jeweils innerhalb eines einzelnen Haushaltsjahres das veranschlagte mehrjährige Gesamtbudget der kambodschanischen ECCC vielfach überschreiten ${ }^{120}$. Für Kambodscha selbst geht es um ein Mindestmaß an später Gerechtigkeit, Aufarbeitung historischer Wahrheit und nicht zuletzt um dringend notwendige Impulse für eine Systemverbesserung im juristischen System insgesamt. Die Herausforderungen sind erheblich, aber es bieten sich Chancen.

Vgl. Hannah Arendt, Eichmann in Jerusalem: A Report on the Banalty of Evil (1977); zur Adaption des Begriffs von der "Banalität des Bösen" auf Kambodscha etwa Hinton (Anm. 5), S. 297 f., argumentierend, dass Befehlsbefolgung allein als Erklärungsmuster für die in Rede stehenden Gewaltexzesse wohl ohnehin nicht genügt.

Die Bedeutung für die Zukunft des Internationalen Strafrechts betont z.B. auch Patricia Wald, Iraq, Cambodia, and International Justice, American University International Law Review 21 (2006), S. 51 (555 f.). 